\title{
MODELOS SEMIPARAMÉTRICOS PARA EVENTOS RECORRENTES
}

\author{
Ângela Tavares Paes
}

\author{
Dissertação apresentada ao \\ Instituto de Matemática e Estatística \\ da Universidade de São Paulo \\ para obtenção do grau de \\ Mestre em Estatística
}

Área de concentração: Estatística

Orientador: Prof. Dr. Antonio Carlos Pedroso de Lima

- São Paulo, dezembro de 1999 - 
Aos meus pais,

que não pouparam esforços para proporcionar meus estudos. 


\section{Sumário}

1 Introdução 1

2 Estimação Não Paramétrica $\quad 5$

2.1 Introdução . . . . . . . . . . . . . . . . . . . . . . 5

2.2 Abordagem de processos de contagem . . . . . . . . . . . . . . . . 6

$2.3 \quad$ O estimador de Nelson-Aalen . . . . . . . . . . . . . . . . . . . 11

2.4 Estimador Produto Limite para uma Matriz de Transição . . . . . . . . . 15

2.4.1 O Estimador de Aalen-Johansen . . . . . . . . . . . . . . . . 18

2.4.2 Representação Martingal e Propriedades . . . . . . . . . . . . . . . 19

2.4.3 Estimadores da matriz de covariância . . . . . . . . . . . . . . 21

2.4 .4 Exemplos . . . . . . . . . . . . . . . . . . . 23

3 Modelos de Regressão $\quad 26$

3.1 Estimação dos parâmetros . . . . . . . . . . . . . . . . . 27

3.1.1 Modelo de Incrementos Independentes (AG) . . . . . . . . . . . 27

3.1.2 Modelo Condicional (PWP) . . . . . . . . . . . . . 30

3.1.3 Modelo Marginal (WLW) . . . . . . . . . . . . . . 32

3.2 Estimação da matriz de probabilidades de transição . . . . . . . . . . . . 37 
3.3 Comentários . . . . . . . . . . . . . . . . . 38

4 Aplicações $\quad 40$

4.1 Estimação sem covariáveis . . . . . . . . . . . . . . . . . . 42

4.2 Comparação de duas amostras . . . . . . . . . . . . . . . . . 45

4.3 Inclusão de covariáveis . . . . . . . . . . . . . . . . . 46

4.4 Considerações finais . . . . . . . . . . . . . . . . . 55

$\begin{array}{ll}\text { A Teoremas } & 56\end{array}$

$\begin{array}{ll}\text { B Aspectos computacionais } & 58\end{array}$

$\begin{array}{ll}\text { C Tabelas complementares } & 63\end{array}$ 


\section{Resumo}

A maioria dos estudos que envolvem Análise de Sobrevivência considera o tempo até a ocorrência de um único evento. Neste trabalho, analisamos situações onde o evento de interesse pode ocorrer mais de uma vez para o mesmo indivíduo. Embora os estudos nessa área tenham recebido considerável atenção nos últimos anos, as técnicas que já existem e que podem ser aplicadas a esses casos especiais ainda são pouco difundidas. O objetivo desta dissertação é descrever alguns métodos estatísticos para análise de eventos recorrentes e discutir suas aplicações. Utilizando a abordagem de processos de contagem multivariados, representamos o problema como um processo de Markov, em que os indivíduos são associados a diferentes estados ao longo do tempo. Esta metodologia consiste em estimar matrizes cujos elementos correspondem às probabilidades de transição entre os estados. Descrevemos métodos de estimação não paramétricos e três modelos semiparamétricos propostos na literatura, baseados no modelo de riscos proporcionais de Cox. Os métodos são ilustrados através de um exemplo baseado em dados reais.

\section{Abstract}

Many studies in life history analysis take into account the time until the occurrence of a terminal event. In this work, we analyse situations where the event of interest is non-fatal and subjects may experience it several times. Although the statistical methods for such purposes have been improved in the last years, the techniques that can be applied in this special cases are not widely disseminated. The objective of this dissertation is to describe some methodologies for the analysis of recurrent events and discuss their applications. Under a multivariate counting processes approach, the problem is represented as Markov processes where the subjects are associated to different states during a follow-up period. The work is concerned with estimating matrices, the elements of which are the transition probabilities between states. We describe nonparametric and semiparametric methods based on the Cox proportional hazards regression model. We compare three models proposed in literature and apply them to a practical example. 


\section{Agradecimentos}

Ao Antonio Carlos, pela orientação, e por ter acreditado desde o início na idéia de transformar uma dúvida de aula em uma dissertação de mestrado.

Ao Júlio César Rodrigues Pereira, grande incentivador e principal responsável pela minha volta à universidade.

Ao Valdir Okano, que foi, sem dúvida, a pessoa que mais colaborou diretamente, viabilizando toda a parte computacional e acompanhando de perto todas as etapas.

Ao Núcleo de Investigações em Nutrição do Instituto de Saúde de São Paulo, em particular à Dóris Lei, por ter fornecido os dados, principal fonte da minha motivação.

Ao Julio Singer, que muito me incentivou desde a graduação e a quem devo grande parte da minha postura profissional.

À Adriana Sañudo, pela amizade e por toda a ajuda, não só nesta fase final como durante todo o curso.

A Helena Teixeira, pela cuidadosa revisão bibliográfica.

Ao Dema, pelo amor, companheirismo, paciência e principalmente, por ter enriquecido tanto a minha vida nestes últimos anos.

Finalmente agradeço a todos os meus amigos, que embora não tenham contribuído diretamente com este trabalho, sempre estiveram ao meu lado, acompanhando com entusiasmo cada etapa e incentivando-me em todos os momentos. 


\section{Capítulo 1}

\section{Introdução}

Em muitos estudos médicos que envolvem o acompanhamento de pacientes, alguns eventos de interesse não são terminais e podem ocorrer mais de uma vez para o mesmo indivíduo (eventos recorrentes). Esta situação é comum em estudos de doenças crônicas, como diabetes, em que o interesse pode ser a ocorrência de hiperglicemias. Outros exemplos freqüentes na literatura são crises de asma, ataques epiléticos, episódios de rejeição em pacientes transplantados, internações hospitalares e infecções de vários tipos.

Diferentemente do caso mais simples, em que se observa um único evento e a partir do momento em que ele acontece, o paciente deixa de ser acompanhado, no caso de eventos recorrentes o paciente continua em observação mesmo depois da ocorrência de cada evento. Como as técnicas usuais não são adequadas para analisar dados deste tipo, muitos pesquisadores consideram como período de observação apenas o tempo até o instante em que o evento ocorre pela primeira vez. Desta forma acabam utilizando somente parte da informação, desperdiçando dados obtidos no resto do acompanhamento.

Diversos artigos têm abordado o problema de eventos recorrentes, sugerindo diferentes metodologias. Lawless \& Nadeaus (1995) utilizaram processos de Poisson para desenvolver modelos que enfocam o número esperado de eventos ocorridos em um determinado intervalo de tempo. Visando facilitar a aplicação desta metodologia a problemas da área médica, Cook (1995) sugere modificações e discute os modelos de Poisson fazendo uma série de considerações sob o ponto de vista clínico. Sob essa mesma abordagem, Cook \& Lawless (1997a) consideram situações em que, além dos eventos recorrentes, existem even- 
tos terminais que impedem a ocorrência dos demais eventos e portanto induzem censuras dependentes.

Glynn, Stukel, Sharp, Bulbolz, Freeman \& Fisher (1993) propõem estimadores para a taxa de ocorrência de eventos recorrentes quando a distribuição do número de eventos para cada indivíduo segue uma distribuição binomial negativa. Stukel, Glynn, Fisher, Sharph, Lu-yao \& Wennberg (1994) generalizam a abordagem proposta por Glynn et al. (1993) incorporando variáveis dependentes do tempo e considerando uma forma arbitrária para a taxa de ocorrência, isto é, sem especificar distribuições.

Várias outras abordagens alternativas têm sido desenvolvidas para tratar de eventos recorrentes (Hoover (1996), Ng \& Cook (1997), Turnbull, Jiang \& Clark (1997) ou Doganaksoy \& Nelson (1998)). Apesar disso, os métodos para a análise de dados desse tipo não estão completamente disseminados e o assunto ainda é alvo de muitas discussões.

Nesta dissertação o problema é tratado sob a abordagem de processos de contagem multivariados. $\mathrm{O}$ desenvolvimento de modelos estatísticos baseados em processos de contagem para analisar dados de sobrevivência foi originalmente introduzido por Aalen (1978) e depois cuidadosamente revisto por Andersen \& Borgan (1985). Esta abordagem nos proporciona ferramentas bastante poderosas capazes de generalizar diversas situações. Além disso, do ponto de vista teórico, a metodologia permite estudar com maior rigor algumas propriedades assintóticas de estimadores não paramétricos e semiparamétricos freqüentemente usados em dados de sobrevivência.

Os exemplos relacionados com doenças crônicas citados no início somente correspondem a eventos imediatamente reversíveis, isto é, em que logo após o evento, o paciente volta ao estado inicial, seja naturalmente ou por intermédio de alguma intervenção médica. Apesar disso, estaremos considerando também eventos temporariamente permanentes, ou seja, aqueles cuja recuperação, quando acontece, leva um certo tempo para se concretizar, fazendo com que o indivíduo permaneça no estado "doente" por um dado período de tempo. Além desses dois casos, a abordagem que utilizamos inclui também outras situações que envolvem, por exemplo, a progressão de uma doença ao longo de diferentes estágios ou ainda eventos terminais, como o óbito.

A idéia é representar o problema como um processo de Markov em que os indivíduos 
vão sendo associados a diferentes estados ao longo do tempo. Assim, é possível estudar as transições entre os estados e estimar as probabilidades de transição de um estado para outro. No caso de eventos com recuperação imediata, a análise se resume a um só tipo de transição. Nos demais, as transições de interesse são definidas segundo a comunicação entre os estados determinada de acordo com a especificação de cada problema.

Vários autores desenvolveram modelos envolvendo processos de Markov. Kay (1986) considera um modelo com vários estados que representam estágios de uma doença e um estado correspondendo ao óbito. Usando métodos paramétricos, ele analisa as transições entre os estados (progressão ou regressão da doença) e o risco de óbito em cada estágio. Marshall \& Jones (1995) generalizam o método de Kay, introduzindo covariáveis e desenvolvem um programa específico para ajustar tais modelos (Marshall, Guo \& Jones 1995). Outros métodos consideram casos muito especiais. Kalbfleisch, Lawless \& Vollmer (1983) por exemplo, analisam modelos markovianos em que só estão disponíveis dados agregados, ou seja, em que, em cada instante avaliado, a única informação é o número de pacientes em cada estado.

Aqui estaremos trabalhando com tempos de observação encarados como valores de uma variável contínua. Além disso, estaremos assumindo que os instantes exatos de transição são conhecidos. Isto às vezes não ocorre na prática. Por exemplo, é muito freqüente que, em estudos de acompanhamento de pacientes, as observações correspondam à visitas periódicas. Desta forma os pacientes são examinados somente na ocasião das visitas, não sendo possível avaliar o que ocorre entre uma visita e outra. Uma aproximação usual consiste em assumir que as mudanças entre estados ocorrem exatamente durante as visitas, porém isso nem sempre é aceitável. Para essas situações alguns autores sugerem que se analisem os dados considerando os intervalos entre visitas (Thall \& Lachin (1988), Thall (1988), Staniswallis, Thall \& Salch (1997)). Neste caso o paciente informa quantos eventos ocorreram desde a sua última visita e a análise é feita considerando o número de eventos por intervalo; quando o paciente falta a uma determinada visita o intervalo é considerado censurado. Uma ressalva é que esses métodos são mais adequados a eventos recorrentes imediatamente reversíveis, pois assumem que o indivíduo volta a estar em risco logo após a ocorrência de cada evento.

Em resumo, considerando que os indivíduos transitam num espaço finito de estados, 
nosso trabalho concentra-se na estimação de matrizes cujos elementos correspondem às probabilidades de transição de um estado para outro. No Capítulo 2 descrevemos métodos de estimação não paramétricos, mais especificamente, o estimador Produto-Limite para a matriz de transição proposto por Aalen \& Johansen (1978). Este estimador é uma generalização do estimador de Kaplan-Meier obtido sob a abordagem de processos estocásticos pontuais.

Para estimar as probabilidades de transição considerando também a influência de covariáveis, consideramos, no Capítulo 3, a metodologia sugerida por Andersen, Hansen \& Keiding (1991), cujos estimadores envolvem parâmetros obtidos por modelos de regressão. Além do modelo de Andersen \& Gill (1982) utilizado como base para esta metodologia, discutimos e comparamos outros dois modelos alternativos desenvolvidos por Prentice, Williams \& Peterson (1981) e por Wei, Lin \& Weissfeld (1989).

Alguns autores também discutem esses mesmos modelos de forma muito interessante (Barai \& Teoh (1997), Wei \& Glidden (1997), Therneau \& Hamilton (1997), Hannan, Shu, Weisdorf \& Goldman (1998)). Baseados em exemplos práticos, eles comparam os métodos enfatizando as aplicações na área médica. Em nosso trabalho, o exemplo de aplicação corresponde a um estudo na área de nutrição desenvolvido no Instituto de Saúde de São Paulo (Capítulo 4). No estudo foram acompanhadas 345 crianças desde o nascimento até dois anos de idade e o principal evento de interesse é o déficit estatural, que pode ocorrer mais de uma vez para uma mesma criança. 


\section{Capítulo 2}

\section{Estimação Não Paramétrica}

Neste capítulo, introduzimos a teoria de processos de contagem e sua utilização para obtenção de estimadores não paramétricos. Consideramos inicialmente o caso mais simples em estudos de sobrevivência em que um único evento é observado, estendendo-o, em seguida, para situações mais genéricas, particularmente aquelas de interesse para eventos recorrentes. São apresentadas propriedades assintóticas para esses estimadores e suas respectivas variâncias.

\subsection{Introdução}

Considere a situação em que $n$ pacientes são observados ao longo do tempo até a ocorrência de algum evento (por exemplo, óbito). Sejam $T_{1}, T_{2}, T_{3}, \ldots, T_{n}$ os tempos de falha desses indivíduos, isto é, os instantes de ocorrência dos eventos, e $C_{1}, C_{2}, C_{3}, \ldots, C_{n}$ seus tempos de censura. A censura geralmente ocorre por delimitação do tempo do estudo, perda de contato com o paciente ou exclusão do indivíduo do estudo por algum motivo (por exemplo, uma intervenção médica).

$\mathrm{Na}$ prática, o que de fato é observado pode ser representado por duas variáveis aleatórias:

$$
\begin{gathered}
Z_{i}=\min \left\{T_{i}, C_{i}\right\} \\
\delta_{i}=I_{\left(T_{i} \leq C_{i}\right)}
\end{gathered}
$$


para $i=1, \ldots, n$, em que $\delta_{i}$ é um indicador de falha, valendo 1 se o indivíduo $i$ falhou durante o período em que estava sendo acompanhado e 0 caso contrário.

\section{Função de risco}

A função de risco é definida pelo limite da probabilidade de o indivíduo falhar no intervalo $[t, t+\Delta t)$ para $\Delta t$ tendendo a zero, dado que ele sobreviveu até o instante $t$, isto é,

$$
\alpha(t)=\lim _{\Delta t \rightarrow 0} \frac{P(t \leq T<t+\Delta t \mid T \geq t)}{\Delta t} .
$$

Esta função pode ser interpretada como a taxa instantânea de falha no tempo $t$. Interessante considerar também a função de risco acumulada dada por

$$
A(t)=\int_{0}^{t} \alpha(s) d s
$$

\section{Função de sobrevivência}

A função de sobrevivência é definida como a probabilidade de um indivíduo não falhar até o instante $t$ e pode ser expressa por

$$
S(t)=P(T>t)=\exp \left(-\int_{0}^{t} \alpha(s) d s\right)=\exp ^{-A(t)}
$$

\subsection{Abordagem de processos de contagem}

Antes de descrever os estimadores não paramétricos, é necessário introduzir alguns conceitos que são exaustivamente utilizados na teoria de processos de contagem. 


\section{Processos de contagem}

Um processo de contagem pode ser entendido como um conjunto de variáveis aleatórias $\{N(t): t \geq 0\}$ tal que:

(i) $N(0)=0$;

(ii) $N(t)<\infty$;

(iii) o processo é contínuo à direita ;

(iv) $N(t)$ tem apenas descontinuidades de tamanho 1 .

O exemplo onde $n$ indivíduos são acompanhados ao longo do tempo até a ocorrência de um evento pode ser convenientemente representado por um processo de contagem multivariado $\mathbf{N}(t)=\left\{N_{1}(t), N_{2}(t), \ldots, N_{n}(t)\right\}$, onde

$$
N_{i}(t)=I_{\left\{Z_{i} \leq t, \delta_{i}=1\right\}} .
$$

Note que o processo $N_{i}(t)$ satisfaz as condições (i)-(iv), pois é zero no instante zero, é finito uma vez que assume apenas os valores 0 e 1 , tem um ponto de descontinuidade de tamanho 1 quando ocorre a falha e é contínuo à direita conforme verificamos na Figura 2.1.

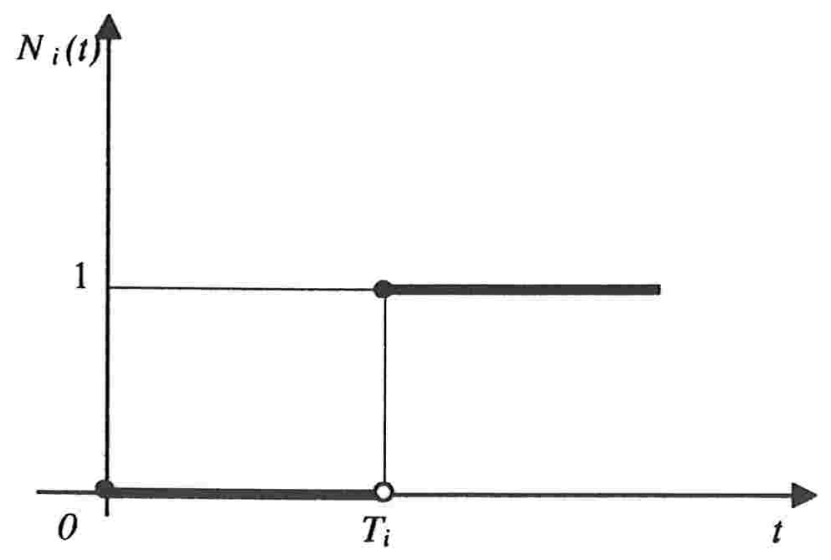

Figura 2.1. Comportamento do processo $N_{i}(t)$ 
Se $N_{i}(t)$ indica que o $i$-ésimo indivíduo falhou até o instante $t$, então

$$
N(t)=\sum_{i=1}^{n} N_{i}(t)
$$

corresponde ao total de falhas até $t$.

Seja $d N(t)=N(t)-N(t-d t)$ o incremento do processo $N(t)$ num intervalo de comprimento $d t$. Observe que $N(t)$ e $N(t-d t)$ só serão diferentes quando houver falha, ou seja,

$$
d N(t)=\left\{\begin{array}{l}
1 \text { se ocorreu falha em t } \\
0 \text { se não ocorreu falha em } \mathrm{t}
\end{array}\right.
$$

Por último, definimos a variável $Y_{i}(t)=I_{\left\{Z_{i} \geq t\right\}}$, que indica se o $i$-ésimo indivíduo está ou não em risco no instante $t$. Assim,

$$
Y(t)=\sum_{i=1}^{n} Y_{i}(t)
$$

representa o total de indivíduos em risco no instante $t$.

\section{Filtragem}

Em linguagem formal, uma filtragem, denotada aqui por $\mathcal{F}^{t^{-}}$, é uma família crescente de sub- $\sigma$-álgebras definidas no espaço amostral. Neste trabalho estaremos nos referindo à filtragem para denotar apenas a história anterior do processo, ou seja, tudo o que aconteceu até $o$ instante imediatamente anterior a $t$.

\section{Processo de intensidade}

$\mathrm{Na}$ teoria de processos de contagem, dizemos que o desenvolvimento no tempo de um processo $N(t)$ é controlado por seu processo de intensidade, $\lambda(t)$. Dada a história anterior, o processo $\lambda(t) d t$ é definido pela probabilidade condicional de que $N(t)$ "salte" em um pequeno intervalo de tempo de comprimento $d t$, isto é, 


$$
\lambda(t) d t \approx P\left(d N(t)=1 \mid \mathcal{F}^{t^{-}}\right)
$$

Note que $P\left(d N(t)=1 \mid \mathcal{F}^{t^{-}}\right)=E\left(d N(t) \mid \mathcal{F}^{t^{-}}\right)$e portanto $\lambda(t)$ pode ser interpretada como a taxa média de troca do processo $N(t)$.

Com base nos conceitos vistos até aqui, podemos escrever informalmente, que a probabilidade de um indivíduo falhar no instante $t$ dado tudo o que ocorreu antes é:

$$
P\left(Z_{i} \in[t, t+d t), \delta_{i}=1 \mid \mathcal{F}^{t^{-}}\right)=\left\{\begin{array}{lll}
\alpha(t) d t & \text { se } & Y_{i}(t)=1 \\
0 & \text { se } & Y_{i}(t)=0
\end{array}\right.
$$

Isto é, a probabilidade instantânea de falha será igual a $\alpha(t) d t$ se o indivíduo estiver em risco e 0 se ele já tiver falhado ou estiver censurado. Observe que uma falha do indivíduo $i$ corresponde a um "salto" do processo $N_{i}(t)$, ou seja, quando $d N_{i}(t)=1$. Isto implica que $P\left(Z_{i} \in[t, t+d t), \delta_{i}=1 \mid \mathcal{F}^{t^{-}}\right)=P\left(d N_{i}(t)=1 \mid \mathcal{F}^{t^{-}}\right)=\alpha(t) d t Y_{i}(t)$ e portanto

$$
\begin{aligned}
E\left(d N(t) \mid \mathcal{F}^{t^{-}}\right) & =P\left(d N(t)=1 \mid \mathcal{F}^{t^{-}}\right) \\
& =\alpha(t) d t \sum_{i=1}^{n} Y_{i}(t) \\
& =\alpha(t) Y(t) d t
\end{aligned}
$$

Com base em (2.1) e (2.2) temos que o processo de intensidade de $N(t)$ é dado por $\lambda(t)=\alpha(t) Y(t)$, o que corresponde ao modelo de intensidade multiplicativo (Aalen 1978).

Da mesma forma que na função de risco acumulada, temos o processo de intensidade acumulado que é dado por

$$
\Lambda(t)=\int_{0}^{t} \lambda(s) d s
$$

O processo de intensidade acumulado representa aproximadamente o número esperado de "saltos" de $N(t)$, isto é, o número esperado de falhas no intervalo $[0, t]$. De fato, pode-se verificar que $\Lambda(t) \cong E(N(t))$. 


\section{Representação Martingal}

Para poder estudar os estimadores de $A(t)$ e $S(t)$, temos que utilizar conceitos básicos da teoria de martingais. Estes conceitos são discutidos com maior rigor em Rogers \& Williams (1994) e Karr (1986). Aqui o maior interesse é entender a idéia sobre o que é um martingal e quais as implicações de suas propriedades. Mais especificamente, estaremos nos concentrando na principal propriedade, que diz que se $M(t)$ é um martingal, $E(M(t))$ | $\left.\mathcal{F}^{s}\right)=E(M(s))$ para todo $s<t$.

Seja $M(t)=N(t)-\Lambda(t)$. Podemos escrever informalmente,

$$
d M(t)=d N(t)-d \Lambda(t)
$$

Usando (2.1) segue que,

$$
\begin{aligned}
E\left(d M(t) \mid \mathcal{F}^{t^{-}}\right) & =E\left((d N(t)-d \Lambda(t)) \mid \mathcal{F}^{t^{-}}\right) \\
& =E\left(d N(t) \mid \mathcal{F}^{t^{-}}\right)-E\left(\lambda(t) d t \mid \mathcal{F}^{t^{-}}\right) \\
& =\lambda(t) d t-\lambda(t) d t=0 .
\end{aligned}
$$

Isto equivale a dizer que a esperança condicional dos incrementos de $M$ em intervalos bem pequenos dada a história anterior desde o início do intervalo é zero. Com base neste fato dizemos que $\Lambda$ é um compensador de $N$ e que $M$ é um martingal. Como $M(0)=0$, temos que $M(t)$ é um martingal de média zero. O processo $N(t)$ escrito como $N(t)=$ $\Lambda(t)+M(t)$ é interpretado como uma decomposição do processo denominada decomposição de Doob Meier.

Este resultado é fundamental para todo o desenvolvimento da teoria, pois permite deduzir propriedades dos estimadores, estimar suas variâncias e posteriormente construir testes utilizando as propriedades assintóticas de martingais expressas através do Teorema de Rebolledo (Rebolledo 1980), conhecido também como Teorema Limite Central para Martingais. 


\subsection{O estimador de Nelson-Aalen}

Para obter heuristicamente um estimador para a função de risco acumulada $A(t)=\int_{0}^{t} \alpha(s) d s$, usamos o fato que $M(t)=N(t)-\Lambda(t)$ é um martingal de média zero.

Seja

$$
M(t)=N(t)-\int_{0}^{t} \lambda(s) d s=N(t)-\int_{0}^{t} \alpha(s) Y(s) d s
$$

Podemos escrever simbolicamente que

$$
\begin{aligned}
& d N(t)=d M(t)+\alpha(t) Y(t) d t \\
& \Rightarrow \alpha(t) d t=\frac{1}{Y(t)}(d N(t)-d M(t)) .
\end{aligned}
$$

Como $M(t)$ é um martingal de média zero, $d M(t)$ pode ser considerado apenas como um "ruído". Portanto um estimador natural de $A(t)$ é:

$$
\hat{A}(t)=\int_{0}^{t} \frac{d N(s)}{Y(s)}
$$

O estimador em (2.3) é chamado estimador de Nelson-Aalen. Andersen, Borgan, Gill \& Keiding (1993) fazem um estudo detalhado sobre este estimador, descrevendo suas propriedades através de aplicações, estudando suas propriedades assintóticas e sugerindo ainda interpretações em termos de máxima verossimilhança.

Através do estimador Nelson-Aalen, podemos obter o estimador Kaplan-Meier (KM) para a função de sobrevivência. Seja $\hat{A}$ o estimador de Nelson-Aalen para a função de risco acumulada. Considerando que os tempos são contínuos, podemos estimar a função de sobrevivência por:

$$
\hat{S}(t)=\underset{s \leq t}{\pi}(1-d \hat{A}(s)) .
$$

em que $\pi$ é chamada integral produto. Este operador é amplamente discutido por Gill \& Johansen (1990), que o estudam com detalhes, considerando sua aplicação em análise 
de sobrevivência. Em nosso trabalho, será suficiente interpretar a integral produto apenas como uma versão contínua do produtório, da mesma forma que a integral pode ser encrada como uma versão contínua da soma.

Se $\hat{A}$ for uma função do tipo escada, podemos escrever:

$$
\hat{S}(t)=\prod_{s \leq t}(1-\Delta \hat{A}(s))=\prod_{s \leq t}\left(1-\frac{\Delta N(s)}{Y(s)}\right)
$$

isto é, a integral produto vira um produto finito considerando apenas os tempos de "saltos" de $N(t)$. Portanto, na prática, caímos no caso discreto, onde o intervalo de tempo é particionado segundo os instantes em que ocorrem falhas. Assim, se $0<T_{1}<T_{2}<\ldots<$ $T_{m} \leq t$ são os tempos de falha ordenados, a função de sobrevivência pode ser estimada por:

$$
\hat{S}(t)=\prod_{i=1}^{m}\left(1-\frac{\Delta N\left(T_{i}\right)}{Y\left(T_{i}\right)}\right),
$$

em que $\Delta N\left(T_{i}\right)=N\left(T_{i}\right)-N\left(T_{i-1}\right)$.

Algumas propriedades que envolvem processos previsíveis, cuja definição formal é dada em Rogers \& Williams (1994), podem ser utilizadas para o estudo do estimador KM. Aqui usaremos basicamente o fato de que todo processo contínuo à esquerda é previsível.

Considere o indicador $J(t)=I_{(Y(t)>0)}$ e sejam

e

$$
A^{*}(t)=\int_{0}^{t} J(s) \alpha(s) d s
$$

$$
S^{*}(t)=\underset{s \leq t}{\prod_{s}}\left(1-d A^{*}(s)\right)
$$

Como as falhas só existem quando há indivíduos em risco, isto é, o processo $N(t)$ só pode "saltar" quando $Y(t)$ for positivo, podemos escrever o estimador de Nelson-Aalen como: 


$$
\hat{A}(t)=\int_{0}^{t} J(s) \frac{d N(s)}{Y(s)} .
$$

Assim, assumindo o modelo de intensidade multiplicativo $(\lambda(t)=\alpha(t) Y(t))$ temos que:

$$
\begin{aligned}
\hat{A}(t)-A^{*}(t) & =\int_{0}^{t} \frac{J(s)}{Y(s)}(d N(s)-d \Lambda(s)) \\
& =\int_{0}^{t} \frac{J(s)}{Y(s)} d M(s) .
\end{aligned}
$$

Reduzindo o Teorema A.1 descrito no Apêndice A ao caso univariado (fazendo $X=$ $\hat{A}, X^{\prime}=A^{*}, Y=\hat{S}$ e $\left.Y^{\prime}=S^{*}\right)$ temos

$$
\begin{aligned}
\frac{\hat{S}(t)}{S^{*}(t)}-1 & =\int_{0}^{t} \hat{S}\left(s^{-}\right)\left(d \hat{A}(s)-d A^{*}(s)\right) S^{*}(s)^{-1} \\
& =\int_{0}^{t} \frac{\hat{S}\left(s^{-}\right)}{S^{*}(s)} \frac{J(s)}{Y(s)} d M(s) .
\end{aligned}
$$

Note que $S\left(s^{-}\right)$é contínua à esquerda e portanto previsível, $J(s)$ e $Y(s)$ são previsíveis e $S^{*}(s)$ depende de uma função determinística e de um processo previsível. Portanto, de acordo com o Teorema A.2, a quantidade expressa em (2.5) corresponde à uma integral estocástica e também é um martingal de média zero.

Este resultado implica que,

$$
E\left(\frac{\hat{S}(t)}{S^{*}(t)}\right)=1
$$

Como $S^{*}(s) \geq S(t)$

$$
E\left(\frac{\hat{S}(t)}{S^{*}(t)}\right) \leq E\left(\frac{\hat{S}(t)}{S(t)}\right) \Rightarrow E(\hat{S}(t)) \geq S(t) .
$$

Portanto, o estimador $\hat{S}(t)$ tende a superestimar $S(t)$. Porém, quando a probabilidade de $Y(t)=0$ é pequena, ou seja, quando quase sempre existirem indivíduos em risco, $S^{*}(t)$ se aproxima de $S(t)$ fazendo com que o viés tenha pouca influência. Assim, dizemos que $\hat{S}(t)$ é um estimador "quase não viciado" para $S(t)$ (Andersen et al. 1993). 


\section{Variância do estimador KM}

Para obter um estimador para a variância de $\hat{S}$, utilizamos o conceito de processo de variação previsível. Definimos o processo de variação previsivel como sendo o processo $\langle M\rangle$ tal que $M^{2}-\langle M\rangle$ é um martingal de média zero. Pode-se verificar que os incrementos de $\langle M\rangle$ correspondem às variâncias condicionais dos incrementos de $M$, isto é,

$$
d\langle M(t)\rangle=\operatorname{Var}\left(d M(t) \mid \mathcal{F}^{t^{-}}\right)=E\left(d M^{2}(t) \mid \mathcal{F}^{t^{-}}\right) .
$$

Como $\frac{\hat{S}(t)}{S^{*}(t)}-1$ é um martingal, usando o fato que $\langle M\rangle$ é um compensador de $M^{2}$ podemos escrever

$$
E\left\{\left(\frac{\hat{S}(t)}{S^{*}(t)}-1\right)^{2}\right\}=E\left\langle\frac{\hat{S}(t)}{S^{*}(t)}-1\right\rangle(t) .
$$

Mas por (2.5) e pela Proposição 2 do Apêndice A,

$$
\begin{aligned}
\left\langle\frac{\hat{S}(t)}{S^{*}(t)}-1\right\rangle(t) & =\left\langle\int_{o}^{t} \frac{\hat{S}\left(s^{-}\right) J(s)}{S^{*}(s) Y(s)} d M(s)\right\rangle \\
& =\int_{0}^{t}\left\{\frac{\hat{S}\left(s^{-}\right) J(s)}{S^{*}(s) Y(s)}\right\}^{2} \lambda(s) \\
& =\int_{0}^{t}\left\{\frac{\hat{S}\left(s^{-}\right)}{S^{*}(s)}\right\}^{2} \frac{J(s)}{Y(s)} \alpha(s) d s .
\end{aligned}
$$

Portanto, considerando que $\hat{S}(t) / S^{*}(t)-1$ é um martingal de média zero, informalmente podemos expressar sua variância por

$$
\operatorname{Var}\left(\frac{\hat{S}(t)}{S^{*}(t)}-1\right)=\int_{0}^{t}\left\{\frac{\hat{S}\left(s^{-}\right)}{S^{*}(s)}\right\}^{2} \frac{J(s)}{Y(s)} d A(s)
$$

Para amostras relativamente grandes, a probabilidade de $Y(t)$ ser zero é pequena, o que faz com que $S^{*}(t) \cong S(t)$ e conseqüentemente 


$$
\operatorname{Var}\left(\frac{\hat{S}(t)}{S^{*}(t)}\right) \cong \operatorname{Var}\left(\frac{\hat{S}(t)}{S(t)}\right)
$$

Substituindo $\hat{S}\left(s^{-}\right)$e $S^{*}(s)$ por $\hat{S}(s)$ (usando a continuidade de $S$ ) e $d A(s)=\alpha(s) d s$ por $d \hat{A}(s)=d N(s) / Y(s)$ chegamos ao seguinte estimador para variância de $\frac{\hat{S}(t)}{S(t)}$ :

$$
\widehat{\operatorname{Var}}\left(\frac{\hat{S}(t)}{S(t)}\right)=\int_{0}^{t} \frac{J(s)}{(Y(s))^{2}} d N(s)
$$

Portanto, um estimador razoável para a variância de $\hat{S}(t)$ para grandes amostras é:

$$
\widehat{\operatorname{Var}}(\hat{S}(t))=(\hat{S}(t))^{2} \int_{0}^{t} \frac{J(s)}{(Y(s))^{2}} d N(s)
$$

que corresponde à conhecida fórmula de Greenwood (Greenwood 1926).

\subsection{Estimador Produto Limite para uma Matriz de Transição}

Até aqui consideramos apenas a situação em que um único evento é observado. Porém, freqüentemente estamos interessados em vários tipos de eventos ou em várias ocorrências de um evento em um mesmo indivíduo.

Situações deste tipo podem ser analisadas sob o ponto de vista de processos de Markov, que consideram que os indivíduos vão sendo associados a estados ao longo do tempo, sendo possível estimar as probabilidades de transição de um estado para outro. Os estados podem ser classificados em absorventes, que uma vez atingido, não permite a transição para outros, e transientes, que permitem a mudança para outros estados.

Suponha que estejamos acompanhando a evolução de $n$ pacientes com interesse em dois eventos: doença e óbito. Uma vez doente, o paciente pode recuperar-se ou morrer, sendo que a morte pode acontecer estando o paciente doente ou não. Neste caso teríamos três 
estados, dois transientes (sadio ou doente) e um absorvente (morto) conforme mostra o esquema a seguir.

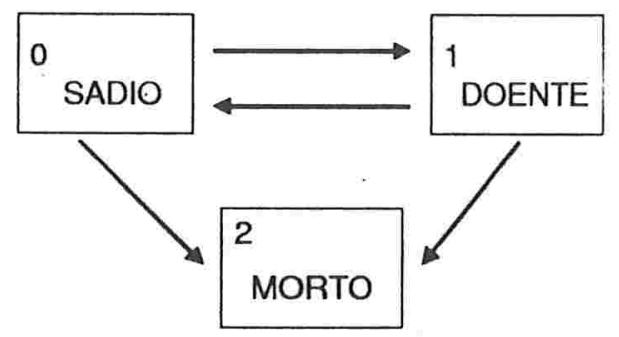

Considerando um processo de Markov com espaço de estados finito $\{1,2, \ldots, k\}$, problemas como esse podem ser representados através do processo de contagem multivariado:

$$
\mathbf{N}(t)=\left\{N_{h j i}(t), h, j=1, \ldots, k \text { e } i=1, \ldots, n\right\}
$$

em que $N_{h j i}(t)$ é o número de vezes que o indivíduo $i$ mudou do estado $h$ para o estado $j$ no intervalo $[0, t]$.

Para cada tipo de transição, temos o processo

$$
N_{h j}(t)=\sum_{i=1}^{n} N_{h j i}(t)
$$

que corresponde ao total de transições diretas de $h$ para $j$ até o instante $t$.

Supondo que duas transições não ocorrem ao mesmo tempo e considerando o incremento do processo $N_{h j}, d N_{h j}(t)=N_{h j}(t)-N_{h j}(t-d t)$, temos o indicador:

$$
d N_{h j}(t)=\left\{\begin{array}{l}
1 \text { se ocorreu transição de } h \text { para } j \text { no instante } t \\
0 \text { caso contrário }
\end{array}\right.
$$

Definimos ainda a variável: 


$$
Y_{h i}(t)= \begin{cases}1 & \begin{array}{l}
\text { se o indivíduo i está no estado } h \text { no instante } \\
\text { imediatamente anterior a } t ;
\end{array} \\
0 & \text { caso contrário } .\end{cases}
$$

Portanto, o processo

$$
Y_{h}(t)=\sum_{i=1}^{n} Y_{h i}(t)
$$

representa o número de indivíduos no estado $h$ no instante $t^{-}$, ou seja, o número de pessoas que no instante $t$ estão em risco de fazer a transição do tipo $h \rightarrow j$, qualquer que seja o estado $j$.

\section{Intensidade de transição}

Neste caso, em lugar da função de risco, definimos as intensidades de transição por:

$$
\alpha_{h j}(t)=\lim _{\Delta t \rightarrow 0} \frac{P_{h j}(t, t+\Delta t)}{\Delta t},
$$

em que $P_{h j}(s, t)$ corresponde à probabilidade de um indivíduo que está no estado $h$ no instante $s$ passar para o estado $j$ no instante $t$.

Portanto, considerando um espaço de estados finito, $\{1,2, \ldots, k\}$, estamos interessados em estimar os elementos de uma matriz $k \times k$ de probabilidades de transição

$$
\mathbf{P}(s, t)=\left\{P_{h j}(s, t)\right\} \text { para } 0 \leq s \leq t
$$

De maneira análoga à que vimos na Seção 2.2, assumimos que o processo $\mathbf{N}(t)$ tem intensidade $\lambda(t)=\left\{\lambda_{h j}(t), h \neq j\right\}$, satisfazendo o modelo de intensidade multiplicativo

$$
\lambda_{h j}(t)=\alpha_{h j}(t) Y_{h}(t)
$$




\subsubsection{O Estimador de Aalen-Johansen}

Nesta seção apresentamos o estimador para uma matriz de transição $\mathbf{P}(s, t)$ proposto por Aalen \& Johansen (1978).

Seja

$$
A_{h j}(t)=\int_{0}^{t} \alpha_{h j}(s) d s, \quad \forall h, j
$$

a função de intensidade de transição acumulada. Definimos $\alpha_{h h}=-\sum_{h \neq j} \alpha_{h j}$ e, conseqüentemente, $A_{h h}=-\sum_{h \neq j} A_{h j}$.

A função matricial $\mathbf{A}=\left\{A_{h j}, h \neq j\right\}$ é denominada medida de intensidade do processo de Markov, onde $A_{h j}$ é chamada função de intensidade acumulada para transições do estado $h$ para o estado $j$ e $A_{h h}$ é a função de intensidade acumulada para transições fora do estado $h$.

Pelo Teorema A.3, temos que a matriz de probabilidades de transição é dada pela integral produto

$$
\mathbf{P}(s, t)=\underset{(s, t]}{\pi}(\mathbf{I}+d \mathbf{A}(u)), \quad \text { onde } \quad \mathbf{A}=\left\{A_{h j}\right\}
$$

Tomando o indicador $J_{h}(t)=I_{\left(Y_{h}(t)>0\right)}$ e o estimador de Nelson-Aalen

$$
\hat{A}_{h j}(t)=\int_{0}^{t} J_{h}(s) \frac{d N_{h j}(s)}{Y_{h}(s)}
$$

um estimador natural para $\mathbf{P}$ é:

$$
\hat{\mathbf{P}}(s, t)=\prod_{(s, t]}(\mathbf{I}+d \hat{\mathbf{A}}(u)) \quad \text { onde } \quad \hat{\mathbf{A}}=\left\{\hat{A}_{h j}\right\} .
$$

Se os estimadores de Nelson-Aalen são funções do tipo escada, o processo é uma cadeia de Markov com tempos discretos e a integral produto torna-se um produto finito de matrizes. Assim, se $T_{1}, T_{2}, \ldots, T_{m}$ são os instantes em que ocorrem transições no intervalo de $s$ a $t$, a matriz $\mathbf{P}$ é estimada por: 


$$
\hat{\mathbf{P}}(s, t)=\prod_{i=1}^{m}\left(\mathbf{I}+\Delta \hat{\mathbf{A}}\left(T_{i}\right)\right)
$$

onde

$$
\Delta \hat{\mathbf{A}}\left(T_{i}\right)= \begin{cases}\frac{\Delta N_{h j}\left(T_{i}\right)}{Y_{h}\left(T_{i}\right)} & \text { para o caso }(h, j) \text { com } h \neq j \\ \frac{-\sum_{h \neq j} \Delta N_{h j}\left(T_{i}\right)}{Y_{h}\left(T_{i}\right)} & \text { para o caso }(h, h) .\end{cases}
$$

Voltando ao caso contínuo, podemos dizer que a matriz de transição é dada por $\mathbf{P}=$ $\pi_{(s, t]} \mathbf{P}(u)$ onde para os intervalos em que não ocorrem transição, a matriz $\mathbf{P}(u)$ em cada instante é estimada pela matriz identidade; em caso contrário, é estimada por $\mathbf{I}+\Delta \hat{\mathbf{A}}\left(T_{i}\right)$.

Este estimador nada mais é do que uma generalização do estimador de Kaplan-Meier, como pode ser verificado no Exemplo 1 da Seção 2.4.4. As propriedades do estimador KM vistas anteriormente também podem ser generalizadas para o estimador Aalen-Johansen conforme discutimos na seção a seguir.

\subsubsection{Representação Martingal e Propriedades}

Assim como para o estimador KM, é possível mostrar que o estimador $\hat{\mathbf{P}}(s, t)$ é "quase não viciado" para $\mathbf{P}(s, t)$. Nesta seção mostramos que isso pode ser feito de maneira análoga à desenvolvida na Seção 2.3 para o estimador $\hat{S}(t)$, porém considerando que agora temos uma matriz no lugar de um escalar.

Definimos

e

$$
A_{h j}^{*}(t)=\int_{0}^{t} J_{h}(u) \alpha_{h j}(u) d u
$$

$$
\mathbf{P}^{*}(s, t)=\underset{(s, t]}{\pi_{(1+}}\left(\mathbf{I}+\mathbf{A}^{*}(u)\right) \quad \text { onde } \quad \mathbf{A}^{*}=\left\{A_{h j}^{*}\right\} .
$$


Utilizando o Teorema A.1 podemos escrever

$$
\begin{aligned}
\hat{\mathbf{P}}(s, t)-\mathbf{P}^{*}(s, t) & =\int_{s}^{t} \underset{[s, u)}{\pi}(\mathbf{I}+d \hat{\mathbf{A}})\left(\hat{\mathbf{A}}(d u)-\mathbf{A}^{*}(d u)\right) \underset{(u, t]}{\pi}\left(\mathbf{I}+d \mathbf{A}^{*}\right) \\
& =\int_{s}^{t} \hat{\mathbf{P}}\left(s, u^{-}\right) d\left(\hat{\mathbf{A}}-\mathbf{A}^{*}\right)(u) \mathbf{P}^{*}(u, t) .
\end{aligned}
$$

Pode-se demonstrar que $\operatorname{det} \mathbf{P}^{*}(s, t)>0$, o que implica que $\mathbf{P}^{*}$ é não singular e portanto admite inversa. Considerando a equação de Chapman-Kolmogorov, $\mathbf{P}(s, t)=$ $\mathbf{P}(s, u) \mathbf{P}(u, t)$, a inversa de $\mathbf{P}^{*}$ pode ser expressa como

$$
\left(\mathbf{P}^{*}(s, t)\right)^{-1}=\left(\mathbf{P}^{*}(s, u) \mathbf{P}^{*}(u, t)\right)^{-1}=\left(\mathbf{P}^{*}(u, t)\right)^{-1}\left(\mathbf{P}^{*}(s, u)\right)^{-1}
$$

Multiplicando os dois lados da equação (2.9) por $\mathrm{P}^{*}(s, t)^{-1}$ temos

$$
\hat{\mathbf{P}}(s, t)\left(\mathbf{P}^{*}(s, t)\right)^{-1}-\mathbf{I}=\int_{s}^{t} \hat{\mathbf{P}}\left(s, u^{-}\right) d\left(\hat{\mathbf{A}}-\mathbf{A}^{*}\right)(u)\left(\mathbf{P}^{*}(s, u)\right)^{-1}
$$

Embora seja trabalhoso, é possível verificar que o elemento $(h, j)$ da matriz $\hat{\mathbf{P}}\left(\mathbf{P}^{*}\right)^{-1}-\mathbf{I}$ tem a forma

$$
\sum_{l=1}^{k} \sum_{m=1}^{k} \int_{s}^{t} \hat{P}_{h l}\left(s, u^{-}\right) P_{m j}^{*}(s, u) d\left(\hat{A}_{l m}-A_{l m}^{*}\right)(u)
$$

em que $\hat{P}_{h l}$ e $P_{m j}^{*}$ são os elementos das matrizes $\hat{\mathbf{P}}$ e $\mathbf{P}^{*}$, respectivamente, nas posições $(h, l)$ e $(m, j)$.

Da mesma forma que na equação (2.4), podemos escrever

$$
\hat{A_{l m}}(t)-A_{l m}^{*}(t)=\int_{0}^{t} \frac{J_{l}(s)}{Y_{l}(s)} d M_{l m}(s)
$$

Como $J_{l}(s)$ e $Y_{l}(s)$ são processos previsíveis e $M_{l m}$ é um martingal de média zero, temos que a matriz $\hat{\mathbf{P}}\left(\mathbf{P}^{*}\right)^{-1}-\mathbf{I}$ é um martingal de média zero, pois corresponde a uma matriz de dimensão $k \times k$ em que todos os elementos são martingais. 
Esta afirmação implica que,

$$
E\left(\hat{\mathbf{P}}(s, t)\left(\mathbf{P}^{*}(s, t)\right)^{-1}\right)=\mathbf{I}
$$

Como comentamos anteriormente, quando a probabilidade de $Y_{h}(u)=0$ é muito pequena, $\mathbf{P}^{*}$ fica próxima de $\mathbf{P}$ o que diminui o viés de $\hat{\mathbf{P}}$. Isto geralmente acontece no caso de amostras grandes, onde a probabilidade de cada um dos estados ficar vazio é menor.

\subsubsection{Estimadores da matriz de covariância}

Para obter a matriz de covariância do estimador da matriz de transição $\mathbf{P}$, usamos a matriz descrita em (2.8). Antes porém, é necessário definir alguns conceitos e fazer uma breve revisão para melhor entender as dimensões do que desejamos estimar.

Estamos interessados em construir a matriz de covariância de uma variável aleatória $\hat{\mathbf{P}}$, matriz de dimensão $k \times k$. Para isso, vamos trabalhar com o operador vec $(\hat{\mathbf{P}})$, cuja função é empilhar as colunas de $\hat{\mathbf{P}}$. Andersen et al. (1993), definem a matriz de covariância de $\hat{\mathbf{P}}$ como a matriz de covariância ordinária de $\operatorname{vec}(\hat{\mathbf{P}})$ dada por

$$
\operatorname{Cov}(\hat{\mathbf{P}})=E\left\{(\operatorname{vec}(\hat{\mathbf{P}})-\operatorname{vec} E(\hat{\mathbf{P}}))(\operatorname{vec}(\hat{\mathbf{P}})-\operatorname{vec} E(\hat{\mathbf{P}}))^{T}\right\}
$$

Como $\hat{\mathbf{P}}$ é uma matriz $k \times k$ e vec $(\hat{\mathbf{P}})$ é um vetor $k^{2} \times 1$, a matriz de covariâncias $\operatorname{Cov}(\hat{\mathbf{P}})$ é uma matriz $k^{2} \times k^{2}$, a saber

$$
\operatorname{Cov}(\hat{\mathbf{P}}(s, t))=\left(\begin{array}{cccccccc}
\sigma_{11}^{2} & \sigma_{11,12} & \ldots & \sigma_{11,1 k} & \ldots & \sigma_{11, k 1} & \ldots & \sigma_{11, k k} \\
\sigma_{12,11} & \sigma_{12}^{2} & \ldots & \sigma_{12,1 k} & \ldots & \sigma_{12, k 1} & \ldots & \sigma_{12, k k} \\
\vdots & \vdots & \ddots & \vdots & \vdots & \vdots & \vdots & \vdots \\
\sigma_{1 k, 11} & \ldots & \ldots & \sigma_{1 k}^{2} & \ldots & \ldots & \ldots & \sigma_{1 k, k k} \\
\vdots & \vdots & \vdots & \vdots & \ddots & \vdots & \vdots & \vdots \\
\sigma_{k 1,11} & \ldots & \ldots & \sigma_{k 1,1 k} & \ldots & \ddots & \ldots & \sigma_{k 1, k k} \\
\vdots & \vdots & \vdots & \vdots & \vdots & \vdots & \ddots & \vdots \\
\sigma_{k k, 11} & \ldots & \ldots & \sigma_{k k, 1 k} & \ldots & \ldots & \ldots & \sigma_{k k}^{2}
\end{array}\right)
$$


em que $\sigma_{h j}^{2}=\operatorname{Var}\left(\hat{P}_{h j}(s, t)\right)$ e $\sigma_{h j, m r}=\operatorname{Cov}\left(\hat{P}_{h j}(s, t), \hat{P}_{m r}(s, t)\right)$.

Note que a matriz $\operatorname{Cov}(\hat{\mathbf{P}})$ pode ser vista como uma composição de $k$ submatrizes quadradas de dimensão $k$, ou seja,

$$
\operatorname{Cov}(\hat{\mathbf{P}}(s, t))=\left(\begin{array}{cccc}
\Sigma_{11} & \Sigma_{12} & \ldots & \Sigma_{1 k} \\
\Sigma_{21} & \Sigma_{22} & \ldots & \Sigma_{2 k} \\
\vdots & \ldots & \ddots & \ldots \\
\Sigma_{k 1} & \Sigma_{k 2} & \ldots & \Sigma_{k k}
\end{array}\right)
$$

em que $\Sigma_{i j}$ corresponde à matriz de covariâncias entre os elementos da $i$-ésima e $j$-ésima coluna de $\hat{\mathbf{P}}$, ou seja, entre $\left(\hat{P}_{i 1}, \hat{P}_{i 2}, \ldots, \hat{P}_{i k}\right)$ e $\left(\hat{P}_{j 1}, \hat{P}_{j 2}, \ldots, \hat{P}_{j k}\right)$.

A partir da definição (2.12), a obtenção do estimador da matriz de covariância de $\hat{\mathbf{P}}$ é análoga à que foi desenvolvida para o estimador KM. Utilizando a Proposição 3 do Apêndice A é possível mostrar que, para grandes amostras, a matriz de covariância da matriz $\hat{\mathbf{P}}(s, t)$ pode ser estimada por

$$
\widehat{\operatorname{Cov}}(\hat{\mathbf{P}}(s, t))=\int_{s}^{t} \hat{\mathbf{P}}(u, t)^{T} \otimes \hat{\mathbf{P}}(s, u) d\left\langle\hat{\mathbf{A}}-\mathbf{A}^{*}\right\rangle(u) \hat{\mathbf{P}}(u, t) \otimes \hat{\mathbf{P}}(s, u)^{T} .
$$

\section{Elementos da matriz de covariância}

A matriz escrita de forma compacta em (2.13), é uma matriz $k^{2} \times k^{2}$ cuja diagonal corresponde às variâncias estimadas dos estimadores $\hat{\mathbf{P}}_{h j}, \forall h, j=1, \ldots, k$. Após alguma manipulação algébrica pode-se obter a forma explícita dos elementos dessa matriz. Assim, o estimador da covariância entre $\hat{P}_{h j}$ e $\hat{P}_{m r}$ pode ser escrito como

$$
\begin{aligned}
\widehat{\operatorname{Cov}}\left(\hat{P}_{h j}(s, t), \hat{P}_{m r}(s, t)\right)= & \sum_{l=1}^{k} \sum_{g \neq l} \int_{s}^{t} \hat{P}_{h g}(s, u) \hat{P}_{m g}(s, u)\left\{\hat{P}_{l j}(u, t)-\hat{P}_{g j}(u, t)\right\} \\
& \quad \times\left\{\hat{P}_{l r}(u, t)-\hat{P}_{g r}(u, t)\right\} J_{g}(u)\left(Y_{g}(u)\right)^{-2} d N_{g l}(u) .
\end{aligned}
$$


É interessante observar que, além dos processos $J_{h}(t)$ e $Y_{h}(t)$, essa covariância envolve os incrementos de $N_{h j}(t)$ referentes a todas as transições possíveis. Note também que a variância dos estimadores $\hat{P}_{h j}$ é obtida tomando-se $m=h$ e $r=j$.

\subsubsection{Exemplos}

Os problemas em estudos de sobrevivência podem ser vistos como processos de Markov com estados a que os indivíduos vão sendo associados ao longo do tempo. O número de estados e a possibilidade de transição entre eles define a dimensão e a forma da matrizfunção $\mathbf{P}(s, t)$ que se pretende estimar. Para entender melhor como essas matrizes são construídas a partir de situações práticas, apresentamos dois exemplos.

Exemplo 1: Considere o caso clássico em análise de sobrevivência onde pacientes em risco são acompanhados até que se observe o óbito. Neste caso temos dois estados, um inicial (em risco) e um absorvente (morto).

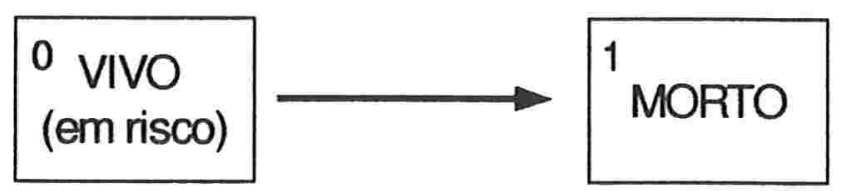

Como a única transição possível é passar do estado 0 para o estado 1 , existem apenas duas probabilidades a serem estimadas: a de permanecer vivo $\left(P_{00}\right)$ e a de morrer $\left(P_{01}\right)$, sendo que uma é complementar da outra. Portanto a matriz de transição estimada para o instante $T_{i}$ é da forma

$$
\mathbf{I}+\Delta \hat{\mathbf{A}}\left(T_{i}\right)=\left(\begin{array}{cc}
1-\frac{\Delta N_{01}\left(T_{i}\right)}{Y_{0}\left(T_{i}\right)} & \frac{\Delta N_{01}\left(T_{i}\right)}{Y_{0}\left(T_{i}\right)} \\
0 & 1
\end{array}\right)
$$


Note que $P_{00}(0, t)$ representa a probabilidade de o indivíduo permanecer vivo até o instante $t$, ou seja, é a função de sobrevivência $S(t)$. De fato, se $T_{1}, T_{2}, \ldots, T_{m}$ são os tempos de falha ordenados,

$$
\hat{P}_{00}(0, t)=\prod_{i=1}^{m}\left(1-\frac{\Delta N_{01}\left(T_{i}\right)}{Y_{0}\left(T_{i}\right)}\right)
$$

que corresponde ao estimador Kaplan-Meier escrito em termos de processos de contagem.

De acordo com a equação (2.14), verificamos que o estimador da variância de $\hat{P}_{00}(0, t)$ pode ser escrito como

$$
\widehat{\operatorname{Var}}\left(\hat{P}_{00}(0, t)\right)=\left\{\hat{P}_{00}(s, t)\right\}^{2} \int_{0}^{t} J_{0}(u)\left(Y_{0}(u)\right)^{-2} d N_{01}(u)
$$

que corresponde à fórmula de Greenwood escrita em termos de processos de contagem.

Exemplo 2: Considere o exemplo dado no início da Seção 2.4.

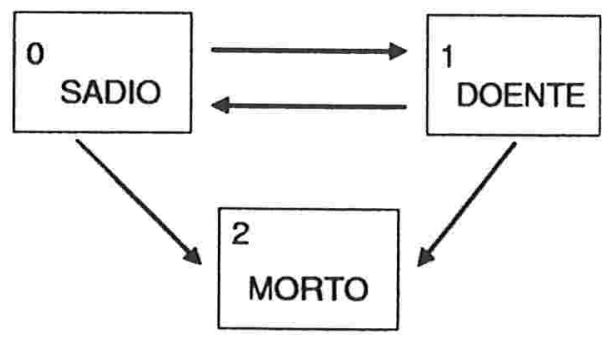

Se $T_{1}, T_{2}, \ldots, T_{m}$ são os instantes em que foram observadas transições no intervalo de $s$ a $t$, a matriz de transição é estimada por

$$
\hat{\mathbf{P}}(s, t)=\prod_{i=1}^{m}\left(\mathrm{I}+\Delta \hat{\mathbf{A}}\left(T_{i}\right)\right)
$$


onde

$$
\mathbf{I}+\Delta \hat{\mathbf{A}}\left(T_{i}\right)=\left(\begin{array}{ccc}
1-\frac{\Delta N_{0}(t)}{Y_{0}(t)} & \frac{\Delta N_{01}(t)}{Y_{0}(t)} & \frac{\Delta N_{02}(t)}{Y_{0}(t)} \\
\frac{\Delta N_{10}(t)}{Y_{1}(t)} & 1-\frac{\Delta N_{1}(t)}{Y_{1}(t)} & \frac{\Delta N_{12}(t)}{Y_{1}(t)} \\
0 & 0 & 1
\end{array}\right)
$$

e $N_{0}\left(T_{i}\right)=N_{01}\left(T_{i}\right)+N_{02}\left(T_{i}\right), N_{1}\left(T_{i}\right)=N_{10}\left(T_{i}\right)+N_{12}\left(T_{i}\right)$.

Neste exemplo, se não houvesse a possibilidade de recuperação o estimador para a matriz de transição no instante $T_{i}$ seria:

$$
\mathbf{I}+\Delta \hat{\mathbf{A}}\left(T_{i}\right)=\left(\begin{array}{ccc}
1-\frac{\Delta N_{0}(t)}{Y_{0}(t)} & \frac{\Delta N_{01}(t)}{Y_{0}(t)} & \frac{\Delta N_{02}(t)}{Y_{0}(t)} \\
0 & 1-\frac{\Delta N_{12}(t)}{Y_{1}(t)} & \frac{\Delta N_{12}(t)}{Y_{1}(t)} \\
0 & 0 & 1
\end{array}\right)
$$

onde $N_{0}\left(T_{i}\right)=N_{01}\left(T_{i}\right)+N_{02}\left(T_{i}\right)$. 


\section{Capitulo 3}

\section{Modelos de Regressão}

No capítulo anterior, o método descrito para estimar as probabilidades de transição considera que a população é homogênea, ou seja, que as probabilidades independem de características individuais representadas por outras variáveis. Na prática, esta é uma suposição pouco razoável, o que torna interessante a construção de modelos que permitam a inclusão de covariáveis usando técnicas de regressão.

Os métodos mais utilizados com essa finalidade são os semiparamétricos, baseados no modelo de riscos proporcionais (Cox 1972). Cox sugere que a função de risco $\alpha(t)$ seja escrita como o produto de uma função de $t$, arbitrária, e de uma função das covariáveis. A forma mais comum para o modelo de Cox é $\alpha(t)=\alpha_{0}(t) \exp \left(\boldsymbol{\beta}^{T} \mathbf{Z}\right)$, onde $\mathbf{Z}$ representa $o$ vetor de covariáveis e $\beta$ o vetor de parâmetros, normalmente desconhecidos.

No caso clássico onde um único evento é observado, os parâmetros de interesse podem ser estimados pelos valores que maximizam a função de verossimilhança parcial (Cox 1975), que na forma de processos de contagem (ver Gill (1984)) pode ser escrita como:

$$
L(\boldsymbol{\beta})=\pi_{t \geq 0} \prod_{i=1}^{n}\left(\frac{Y_{i}(t) \exp \left(\boldsymbol{\beta}^{T} \mathbf{Z}_{i}\right)}{\sum_{j=1}^{n} Y_{j}(t) \exp \left(\boldsymbol{\beta}^{T} \mathbf{Z}_{j}\right)}\right)^{d N_{i}(t)} .
$$

Neste capítulo, descrevemos uma extensão do estimador de Aalen-Johansen (Seção 2.4.1) proposta por Andersen et al. (1991) que permite estimar a matriz de probabilidades 
de transição incorporando a influência de covariáveis. Embora esta metodologia utilize como base o modelo de Andersen \& Gill (1982), consideramos ainda dois modelos alternativos para estimar os parâmetros do modelo de regressão, um sugerido por Prentice et al. (1981) e outro por Wei et al. (1989).

Neste caso, no lugar da função de risco são consideradas as intensidades de transição $\alpha_{h j}(t)$ e em cada modelo são feitas suposições envolvendo, principalmente, a estrutura dos conjuntos de risco.

Como nomenclatura estaremos nos referindo ao modelo de Andersen \& Gill (1982) como Modelo de Incrementos Independentes ou Modelo AG, ao modelo de Prentice et al. (1981) como Modelo Condicional ou PWP e ao modelo desenvolvido por Wei et al. (1989) como Modelo Marginal ou WLW.

\subsection{Estimação dos parâmetros}

\subsubsection{Modelo de Incrementos Independentes (AG)}

O modelo proposto por Andersen \& Gill (1982) considera que cada intensidade de transição é especificada por um modelo de regressão de Cox estratificado com intensidades proporcionais. Para cada indivíduo $i$ com vetor de covariáveis $\mathbf{Z}_{i}^{T}=\left(Z_{i 1}, Z_{i 2}, \ldots, Z_{i p}\right)$, assume-se que a intensidade de transição $h \rightarrow j$ segue o modelo

$$
\alpha_{h j i}(t)=\alpha_{h j 0}(t) \exp \left(\beta_{h j}^{T} \mathbf{Z}_{i}\right)
$$

em que $\alpha_{h j 0}$ é a intensidade de transição $h \rightarrow j$ para indivíduos com $\mathbf{Z}_{i}=\mathbf{0}$ (intensidade padrão) e $\boldsymbol{\beta}_{h j}^{T}=\left(\beta_{h j 1}, \beta_{h j 2}, \ldots, \beta_{h j p}\right)$ é o vetor de parâmetros desconhecidos correspondentes à transição $h \rightarrow j$.

Uma maneira conveniente de reparametrizar esse modelo é considerar um vetor $\beta$ comum a todas as transições e vetores de covariáveis específicos para cada tipo de transição. Por exemplo, no caso de 2 tipos de transição $(0 \rightarrow 1$ e $1 \rightarrow 0)$ e 3 covariáveis $\left(Z_{1}, Z_{2}\right.$ e $\left.Z_{3}\right)$, podemos escrever a expressão $\beta_{h j}^{T} Z_{i}=\beta_{h j 1} Z_{i 1}+\beta_{h j 2} Z_{i 2}+\beta_{h j 3} Z_{i 3}$ como $\boldsymbol{\beta}^{T} \mathbf{Z}_{h j i}$ onde $\boldsymbol{\beta}^{T}=\left(\begin{array}{lllllllllll}\beta_{011} & \beta_{012} & \beta_{013} & \beta_{101} & \beta_{102} & \beta_{103}\end{array}\right) ; \mathbf{Z}_{01 i}^{T}=\left(\begin{array}{llllll}Z_{1 i} & Z_{2 i} & Z_{3 i} & 0 & 0 & 0\end{array}\right) \mathrm{e}$ $\mathbf{Z}_{10 i}^{T}=\left(\begin{array}{lllllll}0 & 0 & 0 & Z_{1 i} & Z_{2 i} & Z_{3 i}\end{array}\right)$. 
Para um modelo com $k$ estados e $p$ covariáveis, consideramos o vetor

$$
\boldsymbol{\beta}^{T}=\left(\beta_{h j l} ; h, j=1, \ldots, k, h \neq j, l=1, \ldots, p\right)
$$

em que $\beta_{h j l}$ é o parâmetro que corresponde à transição do tipo $h \rightarrow j$ referente à covariável $Z_{l}$, e o vetor

$$
\mathbf{Z}_{h j i}= \begin{cases}\mathbf{Z}_{i} & \text { nas posições correspondentes à transição } h \rightarrow j ; \\ 0 & \text { nas demais }\end{cases}
$$

em que $\mathbf{Z}_{i}$ é o vetor de covariáveis associadas ao indivíduo $i$.

Essa reparametrização permite que o modelo (3.1) seja reescrito como:

$$
\alpha_{h j i}=\alpha_{h j 0}(t) \exp \left(\boldsymbol{\beta}^{T} \mathbf{Z}_{h j i}\right)
$$

A partir das observações de $N_{h j i}, Y_{h i}(t)$ e $\mathbf{Z}_{i}$, podemos estimar $\beta$ maximizando a função de verossimilhança parcial de Cox:

$$
L(\boldsymbol{\beta})=\pi \prod_{t} \prod_{h, j, i}\left(\frac{\exp \left(\boldsymbol{\beta}^{T} \mathbf{Z}_{h j i}\right)}{\sum_{l=1}^{n} Y_{h l}(t) \exp \left(\boldsymbol{\beta}^{T} \mathbf{Z}_{h j l}\right)}\right)^{d N_{h j i}(t)}
$$

Do ponto de vista prático, a formulação (3.2) permite o uso de software populares para estimação dos parâmetros, uma vez que é análoga ao modelo de Cox. Do ponto de vista teórico, segundo Andersen et al. (1991), o modelo escrito desta forma facilita a formulação e obtenção das propriedades assintóticas. A normalidade assintótica do estimador de verossimilhança parcial $\hat{\beta}$ pode ser derivada de forma semelhante àquela utilizada pelos métodos clássicos, ou seja, via expansões de Taylor considerando a primeira derivada do $\log L$ em torno do verdadeiro valor do parâmetro $\left(\boldsymbol{\beta}_{0}\right)$. Neste caso, o teorema limite central para martingais (Teorema de Rebolledo) é utilizado no lugar do teorema limite central clássico. 
Derivando o logaritmo da verossimilhança (3.3) em relação a $\beta$ e usando o fato que $d M_{h j i}(u)=d N_{h j i}(u)-\lambda_{h j i}(u) d s$, onde $\lambda_{h j i}(u)$ satisfaz o modelo de intensidade multiplicativo $\left(\lambda_{h j i}(u) d s=\alpha_{h j i}(u) Y_{h i}(u)\right)$, obtemos o vetor escore:

$$
U\left(\boldsymbol{\beta}_{0}, t\right)=\sum_{h, j, i} \int_{0}^{t}\left(\mathbf{z}_{h j i}-\frac{\sum_{l} \mathbf{Z}_{h j l} \lambda_{h j l}(u)}{\sum_{l} \lambda_{h j l}(u)}\right) d M_{h j i}(u)
$$

Como $\mathbf{Z}$ e $\boldsymbol{\lambda}$ são processos previsíveis, $U\left(\boldsymbol{\beta}_{0}, t\right)$ corresponde a uma soma de integrais estocásticas e portanto é um martingal de média zero. Aplicando o teorema de Rebolledo, prova-se que $n^{-1 / 2} U\left(\beta_{0}, t\right)$ converge em distribuição para um processo gaussiano. Em seguida, utilizando a expansão de Taylor é possível mostrar que $n^{1 / 2}\left(\hat{\boldsymbol{\beta}}-\boldsymbol{\beta}_{0}\right) \stackrel{D}{\longrightarrow} N\left(0, \mathbf{I}_{o}^{-1}\left(\boldsymbol{\beta}_{0}\right)\right)$ onde $\mathrm{I}_{o}^{-1}$ é a inversa da matriz de Informação de Fisher observada. Como conseqüência, como $\hat{\boldsymbol{\beta}}$ é consistente, temos que $\hat{\boldsymbol{\beta}} \approx N\left(\boldsymbol{\beta}_{0}, \frac{1}{n} I_{o}^{-1}\left(\boldsymbol{\beta}_{0}\right)\right.$.

\section{Suposições do modelo}

Neste modelo, para cada tipo de transição $h \rightarrow j$, cada indivíduo é tratado como um processo de contagem $N_{h j i}(t)$ cujos incrementos são independentes, ou seja, as recorrências da transição do tipo $h \rightarrow j$ não dependem da história anterior do processo. Isto significa supor que as intensidades de transição $\alpha_{h j}(t)$ não são influenciadas pelo número de transições anteriores (suposição de Markov) nem pelo tempo de permanência nos estados (suposição Semi-Markov). Estas suposições são fortes e muitas vezes na prática podem não estar satisfeitas. Uma tentativa de contornar esse problema seria incluir covariáveis dependentes do tempo que indiquem essas informações no modelo de regressão. Por exemplo, poderíamos incluir uma covariável que indicasse o número de transições anteriores até o instante $t$, ou ainda uma covariável indicando o tempo desde a entrada no estado atual. Desta forma, os testes de significância sobre os parâmetros correspondentes à essas covariáveis indicariam se as suposições são aceitáveis ou não. 


\subsubsection{Modelo Condicional (PWP)}

Em muitas situações práticas, a suposição assumida pelo modelo AG de que as intensidades independem do número de transições anteriores não é razoável. Por exemplo, para algumas doenças infecciosas, à medida que os indivíduos vão tendo infecções, sua capacidade de resposta pode ficar debilitada, fazendo com que a chance de uma nova infecção seja maior. Por outro lado, outras doenças podem trazer uma reação inversa, ou seja, a infecção pode fazer com que o organismo desenvolva anticorpos que aumentem sua defesa e conseqüentemente diminuam o risco de uma nova infeç̧ão. Em ambos os casos, não faz sentido considerar de forma equivalente indivíduos que nunca tiveram a infecção e indivíduos que já foram infectados várias vezes.

Baseados em exemplos como esse, Prentice et al. (1981) consideram importante que além das covariáveis, as intensidades de transição padrão dependam também do número de transições anteriores. Para isso, sugerem que o modelo também seja estratificado segundo uma variável que, ao longo do tempo, separe os indivíduos com uma, duas, três transições e assim por diante.

Considerando que as intensidades de transição podem ser influenciadas também pelo tempo de permanância nos estados, Prentice et al. (1981) sugerem ainda que sejam consideradas duas escalas de tempo, uma desde o início da observação e outra a partir da última transição. Dessa forma, chamando de $t_{N_{h j i}(t)}$ o instante da última transição do tipo $h \rightarrow j$ feita pelo indivíduo $i$ antes do tempo $t$, a intensidade de transição para esse indivíduo segundo os modelos sugeridos pode ser escrita como:

$$
\alpha_{h j s i}(t)=\alpha_{h j s 0}(t) \exp \left(\beta_{h j s}^{T} \mathbf{Z}_{i}\right)
$$

e

$$
\alpha_{h j s i}(t)=\alpha_{h j s 0}\left(t-t_{N_{h j i}(t)}\right) \exp \left(\beta_{h j s}^{T} \mathbf{Z}_{i}\right)
$$

em que $s$ indica a ordem em que a transição ocorre (primeira vez, segunda vez, etc.), $\alpha_{h j s 0}$ e $\boldsymbol{\beta}_{h j s}$ são respectivamente a intensidade padrão e o vetor $p$ x 1 de parâmetros correspondentes à transição $h \rightarrow j$ em relação ao estrato $s$. 
Neste caso temos um vetor de parâmetros para cada tipo de transição, dentro de cada estrato. Da mesma forma que para o modelo AG, podemos reescrever os modelos (3.4) e (3.5) usando covariáveis específicas, isto é considerando um vetor $\beta$ comum e as covariáveis especificadas pelo tipo de transição e agora, também pelo estrato. Assim temos os modelos:

$$
\alpha_{h j s i}(t)=\alpha_{h j s 0}(t) \exp \left(\boldsymbol{\beta}^{T} \mathbf{Z}_{h j s i}\right)
$$

e

$$
\alpha_{h j s i}(t)=\alpha_{h j s 0}\left(t-t_{N_{h j i}(t)}\right) \exp \left(\boldsymbol{\beta}^{T} \mathbf{Z}_{h j s i}\right)
$$

\section{Conjunto de risco}

No modelo condicional, para um determinado tipo de transição, o conjunto de indivíduos em risco de fazer a transição pela segunda vez $(s=2)$ é formado somente pelos indivíduos que já fizeram a transição uma vez, assim como o conjunto de risco para a terceira vez $(s=3)$ contém apenas os indivíduos que fizeram a transição duas vezes. Em resumo, o modelo assume que os indivíduos vão mudando de estratos e só estão em risco de cometer a $s$-ésima transição no instante $t$ aqueles que já fizeram a transição $s-1$ vezes até o tempo $t$.

Portanto, se quisermos escrever a verossimilhança desse modelo de maneira análoga à (3.3) temos que redefinir a variável indicadora de risco como

$$
Y_{h j s i}(t)= \begin{cases}1 & \text { se o indivíduo i está no estado } h \text { no instante anterior a } t \\ \text { e já fez } s-1 \text { vezes a transição } h \rightarrow j & \\ 0 \quad \text { caso contrário }\end{cases}
$$

Assim, a verossimilhança é

$$
L(\boldsymbol{\beta})=\pi \prod_{t} \prod_{h, j, s, i}\left(\frac{\exp \left(\boldsymbol{\beta}^{T} \mathbf{Z}_{h j s i}\right)}{\sum_{l=1}^{n} Y_{h j s l}(t) \exp \left(\boldsymbol{\beta}^{T} \mathbf{Z}_{h j s l}\right)}\right)^{d N_{h j s i}(t)}
$$


Para comparar este resultado com aquele correspondente ao modelo AG, seria necessário supor que não existisse efeito de estrato, ou seja, que $\beta_{h j 1}=\beta_{h j 2}=\beta_{h j 3}=\ldots=\beta_{h j}^{*}$, e estimar um único vetor de parâmetros $\hat{\beta}_{h j}^{*}$. Neste caso, embora as covariáveis não sejam mais especificadas pelo estrato, o modelo continua assumindo que as intensidades de transição padrão de cada estrato são diferentes.

Convém observar que o modelo continua sendo uma generalização do modelo de Cox e que, mesmo com essa reformulação, ainda podem ser utilizados software usuais para estimar os parâmetros.

\subsubsection{Modelo Marginal (WLW)}

No modelo desenvolvido por Wei et al. (1989), a análise de regressão é conduzida considerando tempos de transição multivariados. A distribuição marginal desses tempos é modelada segundo o modelo de riscos proporcionais de Cox.

Assim como no caso anterior, o modelo também é estratificado, sendo o modelo para cada estrato chamado modelo marginal. As intensidades de transição de um indivíduo $i$ são especificadas de forma idêntica a (3.4) para o modelo condicional, isto é, considerando também além do tipo, a ordem em que a transição acontece. A função de verossimilhança parcial do modelo marginal também pode ser escrita como (3.8), o que muda é a definição da variável $Y_{h j s i}$ que indica se o indivíduo $i$ está ou não em risco.

Diferentemente do modelo condicional, o conjunto de risco para a s-ésima transição inclui todos os indivíduos, não necessariamente aqueles que fizeram a transição $s-1$ vezes. Neste caso, em cada tipo de transição $h \rightarrow j$, cada observação corresponde a um vetor de tempos de dimensão $s_{h j} \times 1$ onde $s_{h j}$ é o número máximo de transições do tipo $h \rightarrow j$. Desta forma, os indivíduos que estiverem no estado $h$ são considerados em risco para todas as $s_{h j}$ transições de $h$ para $j$. Por exemplo, em um modelo que considera até 3 transições do tipo $h \rightarrow j$ um indivíduo que faz a transição apenas uma vez e em seguida deixa de ser observado é considerado como uma observação censurada para a segunda e terceira vez, enquanto que no modelo condicional este mesmo indivíduo seria considerado apenas no primeiro estrato.

Portanto, no modelo marginal $Y_{h j s i}$ é definido simplesmente como: 


$$
Y_{h j s i}(t)=\left\{\begin{array}{l}
1 \text { se o indivíduo } i \text { está no estado } h \text { no instante anterior a } t \\
0 \text { caso contrário }
\end{array}\right.
$$

Outra peculiaridade deste modelo ocorre quando os períodos de observação não são contínuos. Isto necessariamente acontece quando estamos estimando probabilidades de transição, pois a partir do momento que o indivíduo é submetido a um tipo de transição, ele sai do conjunto de risco e só volta a ser considerado quando voltar ao mesmo estado. Isto faz com que, ao olharmos um só tipo de transição, cada indivíduo tenha várias descontinuidades do período de observação, o que será considerado como várias censuras. Exemplos destas situações são apresentados com mais detalhes no apêndice de Aspectos Computacionais, onde é discutida também a forma de entrada dos bancos de dados.

\section{Variância robusta}

Sob a suposição de que modelo de Cox é adequado, pode-se mostrar que o estimador de verossimilhança parcial $\hat{\boldsymbol{\beta}}$ é consistente para $\boldsymbol{\beta}$ e que a matriz de covariâncias de $\boldsymbol{\beta}$ pode ser consistentemente estimada por $I_{o}^{-1}(\hat{\boldsymbol{\beta}})$ onde $\mathrm{I}_{o}$ é a matriz de informação de Fisher observada (Andersen \& Gill (1982)).

Segundo Lin \& Wei (1989), mesmo quando há falhas nas suposições do modelo, $\hat{\boldsymbol{\beta}}$ é um bom estimador para $\beta$, porém o mesmo não acontece com a variância, ou seja, neste caso, $\mathbf{I}_{o}^{-1}(\hat{\boldsymbol{\beta}})$ pode não ser um estimador adequado.

O modelo marginal difere dos outros dois principalmente por sugerir um novo estimador para a variância de $\hat{\boldsymbol{\beta}}$, chamada variância robusta. Considerando que a transição $h \rightarrow$ $j$ ocorre até no máximo $s_{h j}$ vezes e tomando $\beta_{h j s}, s=1, \ldots, s_{h j}$ como o vetor $p \times 1$ de parâmetros referente ao s-ésimo modelo marginal, Wei et al. (1989) mostraram que a distribuição conjunta de $\left(\hat{\boldsymbol{\beta}}_{h j 1}, \ldots, \hat{\boldsymbol{\beta}}_{h j s_{h j}}\right)$ pode ser aproximada por uma distribuição normal multivariada com média $\left(\boldsymbol{\beta}_{h j 1}, \ldots, \boldsymbol{\beta}_{h j s_{h j}}\right)$ e matriz de covariância robusta $\mathrm{V}_{h j}$, cuja forma será expressa pelos resíduos leverage derivados por Cain \& Lange (1984), conforme sugerem Therneau \& Hamilton (1997). 
Os resíduos leverage correspondem à uma aproximação das medidas de influência calculadas pela técnica de jackknife (Miller 1974). Estas influências podem ser utilizadas para obter estimadores da variância (variância jackknife) que consistem de uma correção da variância usual no caso de suspeitas sobre a validade do modelo de Cox.

Para explicitar os elementos da matriz de covariância robusta, consideramos inicialmente o modelo marginal para o estrato $s$. Seja $\hat{\boldsymbol{\beta}}_{h j s}$ o vetor obtido pelo método de máxima verossimilhança parcial e $\hat{\boldsymbol{\beta}}_{h j s}^{(i)}$ a estimativa de $\boldsymbol{\beta}_{h j s}$ quando a $i$-ésima observação é retirada da amostra.

O método de Cain \& Lange (1984) considera um vetor escore ponderado $\mathbf{U}(\boldsymbol{\beta}, \mathbf{w})$ em que $\mathbf{w}^{T}=\left(w_{1}, w_{2}, \ldots, w_{n}\right)$ é um vetor $n \times 1$ com pesos atribuídos a cada observação $i$. Supondo que todas as observações têm peso 1 exceto a $i$-ésima, que tem peso 0 , podemos pensar em $\hat{\boldsymbol{\beta}}_{h j s}$ como uma função de $w_{i}$, ou seja, $\hat{\boldsymbol{\beta}}_{h j s}\left(w_{i}\right)=\hat{\boldsymbol{\beta}}_{h j s}$ se $w_{i}=1$ e $\hat{\boldsymbol{\beta}}_{h j s}\left(w_{i}\right)=\hat{\boldsymbol{\beta}}_{h j s}^{(i)}$ se $w_{i}=0$. Utilizando a expansão de Taylor de primeira ordem, a influência da observação $i$, definida por $\hat{\beta}_{h j s}-\hat{\boldsymbol{\beta}}_{h j s}^{(i)}$, pode ser aproximada por

$$
\mathbf{D}_{h j s i}=\left(\frac{\partial \hat{\boldsymbol{\beta}}_{h j s}}{\partial w_{i}}\right)^{T}=\mathbf{L}_{h j s i}\left(\mathbf{A}_{h j s}\right)^{-1}
$$

em que $\mathbf{L}_{h j s i}$ é o vetor 1 x $p$ com os resíduos escores (Fleming \& Harrington 1991) calculados para cada indivíduo e $\mathbf{A}_{h j s}$ é a matriz de covariância usual de dimensão $p \times$, obtida pela inversa da informação de Fisher considerando todo $w_{i}=1, i=1, \ldots, n$.

Se $\mathbf{L}_{h j s}$ é a matriz $n \times p$ de escores residuais composta pela combinação dos vetores $\mathbf{L}_{h j s i}$, a matriz dos resíduos leverage definida por $\mathbf{D}_{h j s}=\mathbf{L}_{h j s}\left(\mathbf{A}_{h j s}\right)^{-1}$ pode ser interpretada como uma medida de influência que considera os resíduos do ajuste (resíduos martingais), a discrepância dos valores das covariáveis e a variabilidade das estimativas.

Cain \& Lange (1984) comentam que esta aproximação é a mesma usada para obtenção do jackknife infinitesimal (Miller 1974). Portanto, se $D_{h j s m i}$ é a influência da $i$-ésima observação no parâmetro $\hat{\beta}_{h j s m}$, a covariância assintótica jackknife entre os parâmetros $\hat{\beta}_{h j s m}$ e $\hat{\beta}_{h j s l}$ pode ser aproximadamente estimada por $\sum_{i=1}^{n} D_{h j s m i} D_{h j s l i}$. Generalizando para todos os modelos marginais temos que o estimador da matriz de covariância robusta $\operatorname{de} \hat{\boldsymbol{\beta}}_{h j}=\left(\hat{\boldsymbol{\beta}}_{h j 1}, \ldots, \hat{\boldsymbol{\beta}}_{h j s_{h j}}\right)^{T}$ pode ser expresso por 


$$
\hat{\mathbf{V}}_{h j}=\left(\begin{array}{ccccc}
\hat{\mathbf{V}}_{h j 11} & \hat{\mathbf{V}}_{h j 12} & \ldots & \ldots & \hat{\mathbf{V}}_{h j 1 s_{h j}} \\
\hat{\mathbf{V}}_{h j 21} & \hat{\mathbf{V}}_{h j 22} & \ldots & \ldots & \hat{\mathbf{V}}_{h j 2 s_{h j}} \\
\vdots & \vdots & \ddots & & \vdots \\
\vdots & \vdots & & \ddots & \vdots \\
\hat{\mathbf{V}}_{h j s_{h j} 1} & \hat{\mathbf{V}}_{h j s_{h j} 2} & \ldots & \ldots & \hat{\mathbf{V}}_{h j s_{h j} s_{h j}}
\end{array}\right)
$$

em que cada elemento é a matriz $\hat{\mathbf{V}}_{h j l c}=\mathbf{D}_{h j l}^{T} \mathbf{D}_{h j c}$ de dimensão $p \times p$.

Lin \& Wei (1989) concluem através de estudos empíricos que o estimador $\hat{\mathbf{V}}_{h j}$ é robusto em relação a violações do modelo de Cox, seja na falta de proporcionalidade, na omissão de covariáveis importantes ou até mesmo na forma da função das covariáveis.

$\mathrm{Na}$ prática, estaremos mais interessados nos elementos da diagonal principal da matriz $\hat{\mathbf{V}}_{h j}$, que correspondem às variâncias robustas estimadas de cada parâmetro do modelo. Podemos também inverter a ordem dos parâmetros e considerar as submatrizes da diagonal de $\hat{\mathbf{V}}_{h j}$ como sendo as matrizes de covariância de dimensão $s_{h j} \times s_{h j}$ em relação à cada covariável, no lugar de considerar as matrizes $p \times p$ de cada modelo marginal.

É interessante observar que a matriz de covariância de cada modelo marginal $\hat{\mathbf{V}}_{h j s}$ pode ser escrita como $\mathbf{A}_{h j s}^{-1} \mathbf{B}_{h j s} \mathbf{A}_{h j s}^{-1}$ onde $\mathbf{B}_{h j s}=\mathbf{L}_{h j s}^{T} \mathbf{L}_{h j s}$. Desta forma, este estimador é semelhante ao estimador "sanduíche" estudado no caso paramétrico, definido por

$$
\hat{V}(\hat{\boldsymbol{\beta}})=\mathbf{I}_{o}^{-1}(\hat{\boldsymbol{\beta}}) \hat{\mathbf{B}}(\hat{\boldsymbol{\beta}}) \mathbf{I}_{o}^{-1}(\hat{\boldsymbol{\beta}}),
$$

em que $\hat{\mathbf{B}}(\boldsymbol{\beta})=n^{-1} \sum \mathbf{U}_{i}(\boldsymbol{\beta}) \mathbf{U}_{i}^{T}(\boldsymbol{\beta})$ e $\mathbf{U}_{i}(\boldsymbol{\beta})$ é a contribuição da $i$-ésima observação para a função escore. Porém, neste caso, a forma da matriz $\hat{\mathbf{B}}(\boldsymbol{\beta})$ é mais complicada, uma vez que a função escore não é mais uma soma de $n$ vetores aleatórios independentes e identicamente distribuídos.

\section{Teste de hipótese}

Para avaliar a influência das covariáveis, é possível usar para cada uma delas uma única estatística que sirva para testar o efeito dos parâmetros de todos os modelos marginais conjuntamente. Seja $\hat{\boldsymbol{\beta}}_{h j m}=\left(\hat{\beta}_{h j 1 m}, \hat{\beta}_{h j 2 m}, \ldots, \hat{\beta}_{h j s_{h j} m}\right)^{T}$ o vetor dos parâmetros estimados 
para a covariável $m$ em relação à transição $h \rightarrow j$. Considerando $\hat{\mathrm{V}}_{h j m}$ como a matriz de covariância estimada $s_{h j} \times s_{h j}$ correspondente a $\hat{\boldsymbol{\beta}}_{h j m}$, Wei et al. (1989) sugerem que a hipótese $H_{0}: \hat{\beta}_{h j 1 m}=\hat{\beta}_{h j 2 m}=\ldots=\hat{\beta}_{h j s_{h j} m}=0$ seja testada através da estatística de Wald

$$
W=\hat{\beta}_{h j m}^{T} \hat{\mathbf{V}}_{h j m}^{-1} \hat{\beta}_{h j m}
$$

cuja distribuição assintótica é qui-quadrado com $s_{h j}$ graus de liberdade.

\section{Estimador geral}

Assim como no modelo condicional, neste modelo estimamos vários parâmetros para cada covariável. No caso de haver interesse e possibilidade de estimar um único vetor de parâmetros, Wei et al. (1989) sugerem que este parâmetro comum seja estimado por uma combinação linear dos parâmetros de cada modelo marginal.

Supondo que para a covariável $m, \beta_{h j 1 m}=\beta_{h j 2 m}=\ldots=\beta_{h j s_{h j} m}=\beta_{h j m}^{*}$, podemos estimar $\beta_{h j m}^{*}$ por

$$
\hat{\beta}_{h j m}^{*}=\sum_{k=1}^{s_{h j}} c_{h j k m} \hat{\beta}_{h j k m},
$$

$\operatorname{com} \sum_{k=1}^{s_{h j}} c_{h j k m}=1$.

Se $\mathbf{V}_{h j m}$ é a matriz de covariância associada à covariável $m$, segundo Wei \& Johnson (1985) é possível mostrar que o estimador com pesos

$$
\mathbf{c}_{h j m}=\left(c_{h j 1 m}, \ldots, c_{h j s_{h j} m}\right)^{T}=\left(\mathbf{e}^{T} \hat{\mathbf{V}}_{h j m}^{-1} \mathbf{e}\right)^{-1} \hat{\mathbf{V}}_{h j m}^{-1} \mathbf{e}
$$

em que e é um vetor com todos os elementos iguais a 1, é aquele que tem a menor variância assintótica entre todos os estimadores lineares.

Como conseqüência, temos que a matriz de covariância de $\hat{\beta}_{h j m}^{*}$ pode ser estimada por $\hat{V}_{h j m}^{*}=\mathbf{c}_{h j m}^{T} \hat{\mathbf{V}}_{h j m} \mathbf{c}_{h j m}$. Assim, a significância do parâmetro comum pode ser testada através da estatística

$$
\frac{\left\{\hat{\beta}_{h j m}^{*}\right\}^{2}}{\hat{V}_{h j m}^{*}} \stackrel{a}{\sim} \chi_{1}^{2} .
$$




\subsection{Estimação da matriz de probabilidades de tran- sição}

Estimados os parâmetros, podemos agora estimar a matriz de probabilidades de transição considerando a presença de covariáveis. Este estimador corresponde a uma generalização do estimador de Aalen-Johansen visto no Capítulo 2 com a diferença que as intensidades de transição acumuladas são expressas também pelos parâmetros do modelo de regressão.

Para estimar as intensidades de transição integradas padrão, $\mathbf{A}_{h j 0}(t)=\int_{0}^{t} \alpha_{h j 0}(u) d u$, podemos usar os estimadores de Nelson-Aalen:

$$
\hat{A}_{h j 0}(t ; \hat{\boldsymbol{\beta}})=\int_{0}^{t} \frac{J_{h}(u)}{\sum_{l=1}^{n} Y_{h l}(u) \exp \left(\boldsymbol{\beta}^{T} \mathbf{Z}_{h j l}\right)} d N_{h j}(u)
$$

$h, j=1, \ldots, k ; h \neq j$.

A partir dessas estimativas, podemos estimar as probabilidades de transição $P_{h j}\left(s, t ; \mathbf{Z}_{0}\right)$ para indivíduos com um particular vetor de covariáveis $\mathbf{Z}_{0}$.

Se $\mathbf{Z}_{h j 0}$ é o vetor de covariáveis específicas correspondente a $\mathbf{Z}_{0}$, tomando

$$
\hat{A}_{h j}\left(t ; \mathbf{Z}_{0}\right)=\hat{A}_{h j 0}(t ; \hat{\boldsymbol{\beta}}) \exp \left(\boldsymbol{\beta}^{T} \mathbf{Z}_{h j 0}\right), \quad h \neq j
$$

e

$$
\hat{A}_{h h}\left(t ; \mathbf{Z}_{0}\right)=-\sum_{h \neq j} \hat{A}_{h j}\left(t ; \mathbf{Z}_{0}\right), \quad h=1, \ldots, k
$$

a matriz $k \times k$ de probabilidades de transição pode ser estimada por

$$
\hat{\mathbf{P}}\left(s, t ; \mathbf{Z}_{0}\right)=\underset{(s, t]}{\pi_{1}}\left(\mathbf{I}+d \hat{\mathbf{A}}\left(u ; \mathbf{Z}_{0}\right)\right)
$$

Andersen et al. (1991) mostram que as propriedades assintóticas e a matriz de covariância de $\hat{\mathbf{P}}(s, t, \mathbf{Z})$ podem ser obtidas de maneira análoga à metodologia descrita na Seção 2.4.3 do Capítulo 2. 
Como já comentamos anteriormente, na prática acabamos trabalhando com tempos discretos e a integral produto em (3.9) transforma-se em um produto finito de matrizes. Portanto, se $T_{1}, T_{2}, \ldots, T_{m}$ são os instantes em que são observadas as transições no período de $s$ a $t$, para um determinado vetor $\mathbf{Z}_{0}$ a matriz $\hat{\mathbf{P}}$ é dada por

$$
\hat{\mathbf{P}}\left(s, t ; \mathbf{Z}_{0}\right)=\prod_{i=1}^{m}\left(\mathbf{I}+\Delta \hat{\mathbf{A}}\left(T_{i}, \mathbf{Z}_{0}\right)\right)
$$

onde

$$
\Delta \hat{\mathbf{A}}\left(T_{i}, \mathbf{Z}_{0}\right)= \begin{cases}\frac{J_{h}\left(T_{i}\right) \Delta N_{h j}\left(T_{i}\right) \exp \left(\boldsymbol{\beta}^{T} \mathbf{Z}_{h j}\right)}{\sum_{l=1}^{n} Y_{h l}\left(T_{i}\right) \exp \left(\beta^{T} \mathbf{Z}_{h j l}\right)} & \text { para } h \neq j ; \\ -\sum_{h \neq j} \frac{J_{h}\left(T_{i}\right) \Delta N_{h j}\left(T_{i}\right) \exp \left(\beta^{T} \mathbf{Z}_{h j 0}\right)}{\sum_{l=1}^{n} Y_{h l}\left(T_{i}\right) \exp \left(\boldsymbol{\beta}^{T} \mathbf{Z}_{h j l}\right)} & \text { para } h=j .\end{cases}
$$

Convém observar que este estimador não é mais uma generalização do estimador usual, isto é, no caso de um processo com dois estados (0-vivo e 1-morto), $\hat{\mathbf{P}}_{00}\left(0, t ; \mathbf{Z}_{0}\right)$ não corresponde a exatamente o estimador da função de sobrevivência $S\left(t, \mathbf{Z}_{0}\right)=e^{A_{010}(t) \exp \left(\hat{\boldsymbol{\beta}}(t) \mathbf{Z}_{0}\right)}$ usualmente obtido pelo modelo de Cox. Enquanto $S\left(t, \mathbf{Z}_{0}\right)$ é sempre um valor não-negativo, $\hat{\mathbf{P}}_{00}$ pode até assumir valores negativos caso haja uma extrapolação para conjuntos de risco muito pequenos.

\subsection{Comentários}

O aspecto mais importante a ser comentado é o fato de que nenhum dos modelos apresentados impõe uma estrutura específica de correlação entre as observações de um mesmo indivíduo. Embora o modelo marginal considere isso de uma certa forma, a dependência entre os tempos de falha é tratada como nuisance.

Alguns modelos têm sido desenvolvidos para considerar essas correlações explicitamente sob o enfoque do modelo de Cox. Esta modelagem é concretizada através de modelos de fragilidade ou frailty models (Cai, Sen \& Zhou 1999), onde a "fragilidade" corresponde à um efeito aleatório que induz a dependência entre os tempos de falha. Condicionalmente a esse efeito aleatório, para cada indivíduo, a intensidade de transição segue um modelo de riscos proporcionais. 
Apesar de os modelos de fragilidade serem intuitivamente mais adequados, a modelagem é bem mais complexa e envolve um número maior de parâmetros, além de serem mais difíceis de implementar computacionalmente. Além disso, apesar de a teoria assintótica ter sido desenvolvida por Murphy $(1994,1995)$, tem sido observada grande instabilidade nos testes usualmente empregados.

Outro fator a ser considerado é a estrutura dos conjuntos de risco. Segundo Cook \& Lawless (1997b), em algumas situações, a estrutura imposta pelo modelo marginal pode parecer inconsistente. Por exemplo, em casos onde a ordem da ocorrência dos eventos é importante, pode não haver sentido considerar que os indivíduos estão em risco de fazer determinada transição sem ter feito as anteriores.

Nesses modelos estamos supondo ainda a ausência de interações entre as covariáveis e a proporcionalidade entre as intensidades de transição. Os métodos tradicionais para checar estas suposições são conhecidos e podem ser adaptados também à esses modelos. Sendo assim, a existência de termos de interação pode ser avaliada através de um teste da razão de verossimilhanças parcial e a suposição de proporcionalidade pode ser verificada graficamente, considerando os estimadores de Nelson-Aalen para diferentes estratos ou então introduzindo no modelo a covariável $Z \cdot \log (t / 1000)$ onde $Z$ é a covariável cuja proporcionalidade deve ser testada. 


\section{Capítulo 4}

\section{Aplicações}

O conjunto de dados que iremos analisar corresponde a um estudo feito pelo Núcleo de Investigação em Nutrição do Instituto de Saúde de São Paulo em 1997. O objetivo geral do trabalho era estudar o crescimento de crianças entre o nascimento e os 2 anos de vida, enfocando principalmente o retardo do crescimento físico (déficit estatural) que segundo Gopalan (1988) é um fator altamente correlacionado com a desnutrição.

A amostra era constituída por 345 crianças matriculadas em um programa de suplementação alimentar em 13 unidades de saúde no município de Osasco. Foram incluídas no estudo apenas as crianças que haviam nascido com peso superior a $2500 \mathrm{~g}$ e que tinham pelo menos um irmão com menos de cinco anos.

$\mathrm{Na}$ ocasião da matrícula foram registradas as seguintes variáveis:

- Data de nascimento;

- Peso ao nascer (em gramas x 100);

- Sexo;

- Altura da mãe (em cm);

- Altura dos irmãos (em cm);

- Número de irmãos menores de 7 anos (Um, Dois, Três ou mais);

- Área geográfica de moradia (Central ou Periférica); 
As crianças foram acompanhadas desde o nascimento até os 2 anos de idade, sendo avaliadas mensalmente durante o primeiro ano e trimestralmente no segundo ano. Cada criança fez no máximo 17 visitas nas quais eram registradas a data e a altura da criança $(\mathrm{em} \mathrm{cm})$.

\section{Déficit estatural}

Foram consideradas com déficit estatural as crianças cujo índice estatura/idade era inferior a -2 Z escores da mediana esperada pelo National Center for Health Statistics, segundo recomendação da Organização Mundial de Saúde. Como esta classificação foi definida apenas no banco de dados ao final da coleta, os agentes de saúde que faziam as medidas não podiam avaliar, durante a medição, se a criança estava ou não deficitária, não havendo portanto nenhum tipo de intervenção visando a recuperação da criança no caso de déficit.

Um dos objetivos específicos era saber se o fato de já existir um irmão com déficit estatural aumentaria a probabilidade de a criança tornar-se deficitária. A partir da altura dos irmãos e utilizando o mesmo critério de classificação (com ou sem déficit estatural) criamos uma nova variável indicando o estado da família na época do nascimento da criança (1-com filhos deficitários, 0-sem filhos deficitários).

As idades das crianças em cada visita foram calculadas, em meses, através da diferença entre a data da visita e a data de nascimento. Apesar da orientação para que as visitas fossem feitas de mês em mês e depois a cada três meses, na prática era impossível forçar que as datas coincidissem exatamente com esses períodos. Portanto, embora próximas, as idades de avaliação não eram as mesmas para todas as crianças.

Resumindo, as variáveis de fato consideradas na análise foram a idade, que corresponde à escala de tempo com origem dada pelo nascimento da criança; peso ao nascer, sexo, altura da mãe, número de irmãos menores de 7 anos, área geográfica e estado da família (variáveis independentes do tempo referentes ao período inicial) e o déficit estatural (0ausente e 1-presente), variável medida ao longo do tempo. 


\subsection{Estimação sem covariáveis}

Uma criança pode estar abaixo da estatura esperada em uma determinada visita, crescer naturalmente e recuperar-se, depois retardar o crescimento e ficar deficitária novamente e assim por diante. Portanto cada criança pode estar deficitária mais que uma vez fazendo com que o evento de interesse (déficit estatural) seja recorrente. Neste caso, temos um processo de Markov com dois estados (1-com déficit e 0-sem déficit) conforme mostra o diagrama a seguir:

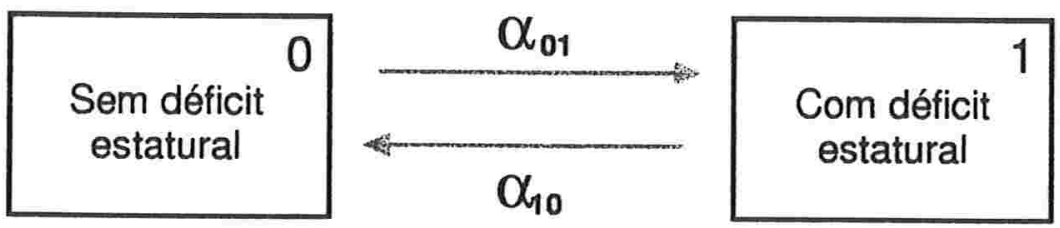

Como as avaliações não eram feitas continuamente e sim em visitas sucessivas, não era possível avaliar o instante exato da passagem de um estado para outro. A transição só era percebida quando o estado da visita atual era diferente do estado da visita anterior e sabia-se apenas que havia ocorrido uma mudança no período entre as visitas. Apesar disso, para utilização da metodologia faremos a suposição de que as transições ocorreram exatamente na data da visita em que foi observada a mudança de estados. Vale lembrar que os instantes referidos aqui correspondem às idades das crianças na ocasião das visitas.

Assim, se $0<T_{1}, T_{2}, \ldots, T_{m} \leq t$ são os tempos em que foram observadas transições no intervalo $(0, t]$, a matriz de transição é estimada por

$$
\hat{\mathbf{P}}(0, t)=\prod_{i=1}^{m}\left(\mathbf{I}+\Delta \hat{\mathbf{A}}\left(T_{i}\right)\right),
$$

em que

$$
\mathbf{I}+\Delta \hat{\mathbf{A}}\left(T_{i}\right)=\left(\begin{array}{cc}
1-\frac{\Delta N_{01}\left(T_{i}\right)}{Y_{0}\left(T_{i}\right)} & \frac{\Delta N_{01}\left(T_{i}\right)}{Y_{0}\left(T_{i}\right)} \\
\frac{\Delta N_{10}\left(T_{i}\right)}{Y_{1}\left(T_{i}\right)} & 1-\frac{\Delta N_{10}\left(T_{i}\right)}{Y_{1}\left(T_{i}\right)}
\end{array}\right)
$$


$\Delta N_{01}\left(T_{i}\right)$ é o número de crianças que se tornaram deficitárias na idade $T_{i}$;

$\Delta N_{10}\left(T_{i}\right)$ é o número de crianças que se recuperaram na idade $T_{i}$;

$Y_{1}\left(T_{i}\right)$ e $Y_{0}\left(T_{i}\right)$ são respectivamente o número de crianças com e sem déficit estatural na idade $T_{i}$

Desta forma, obtemos uma matriz $2 \times 2$ dada em função do tempo com as probabilidades de transição e de permanência nos estados estimadas, isto é,

$$
\hat{\mathbf{P}}(0, t)=\left(\begin{array}{ll}
\hat{P}_{00}(0, t) & \hat{P}_{01}(0, t) \\
\hat{P}_{10}(0, t) & \hat{P}_{11}(0, t)
\end{array}\right)
$$

Utilizando a expressão (2.14) e considerando que $T_{1}, T_{2}, \ldots, T_{m}$ são os instantes em que ocorrem transições no intervalo $(0, t]$, o estimador da variância de $\hat{P}_{01}(t)$ pode ser escrito como

$$
\begin{aligned}
\widehat{\operatorname{Var}} \hat{P}_{01}(0, t) & =\sum_{i=1}^{m}\left(\hat{P}_{00}\left(0, T_{i}\right)\right)^{2}\left\{\hat{P}_{11}\left(T_{i}, T_{m}\right)-\hat{P}_{01}\left(T_{i}, T_{m}\right)\right\}^{2} J_{0}\left(T_{i}\right)\left(Y_{0}\left(T_{i}\right)\right)^{-2} d N_{01}\left(T_{i}\right) \\
& +\sum_{i=1}^{m}\left(\hat{P}_{01}\left(0, T_{i}\right)\right)^{2}\left\{\hat{P}_{01}\left(T_{i}, T_{m}\right)-\hat{P}_{11}\left(T_{i}, T_{m}\right)\right\}^{2} J_{1}\left(T_{i}\right)\left(Y_{1}\left(T_{i}\right)\right)^{-2} d N_{10}\left(T_{i}\right)
\end{aligned}
$$

A variância estimada de $\hat{P}_{10}(t)$ pode ser obtida pela mesma expressão substituindo 0 por 1 e vice-versa. 


\section{Resultados}

O Gráfico 4.1 apresenta as probabilidades de déficit estatural $\left(P_{01}\right)$ e de recuperação $\left(P_{10}\right)$ ao longo do tempo. Embora essas probabilidades não sejam exatamente complementares elas estão relacionadas uma vez que existe uma relação direta entre os conjuntos de risco $Y_{0}\left(T_{i}\right)$ e $Y_{1}\left(T_{i}\right)$ utilizados para calcular as taxas de transição.

Apesar de algumas crianças terem sido acompanhadas até quase 27 meses de idade, o gráfico considera apenas os primeiros 24 meses, pois a partir daí o número de crianças em risco torna-se bastante reduzido.

Observamos que, em geral, a probabilidade de uma criança se recuperar de um estado de déficit é consideravelmente maior do que a probabilidade de tornar-se deficitária, principalmente a partir dos dois meses de idade. Aparentemente a idade mais crítica é aos 20 meses quando a probabilidade de déficit estatural chega a aproximadamente $33 \%$.

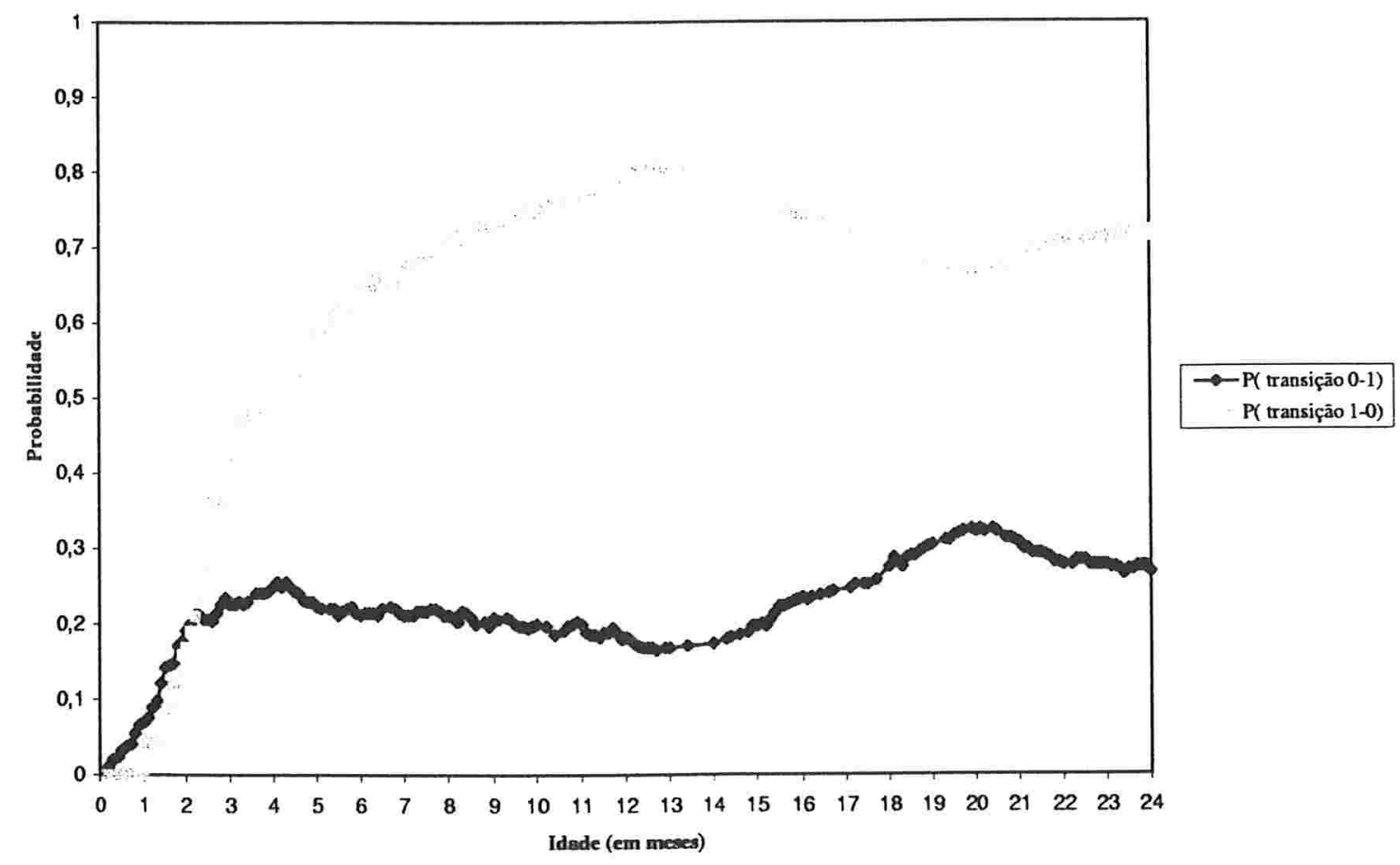

Gráfico 4.1: Probabilidade de déficit estatural e de recuperação 


\subsection{Comparação de duas amostras}

Como já dissemos, um dos objetivos era saber se crianças nascidas em famílias que já tinham outros filhos deficitários tinham maior risco de déficit estatural do que crianças de famílias cujos filhos não eram deficitários. Para isso dividimos a amostra em dois grupos segundo a condição da família e estimamos as probabilidades da mesma forma que na seção anterior. A seguir apresentamos o gráfico com as probabilidades estimadas de déficit estatural $\left(\hat{P_{01}}\right)$ e seus respectivos intervalos de confiança $(95 \%)$ calculados a partir das variâncias dadas em (4.1).

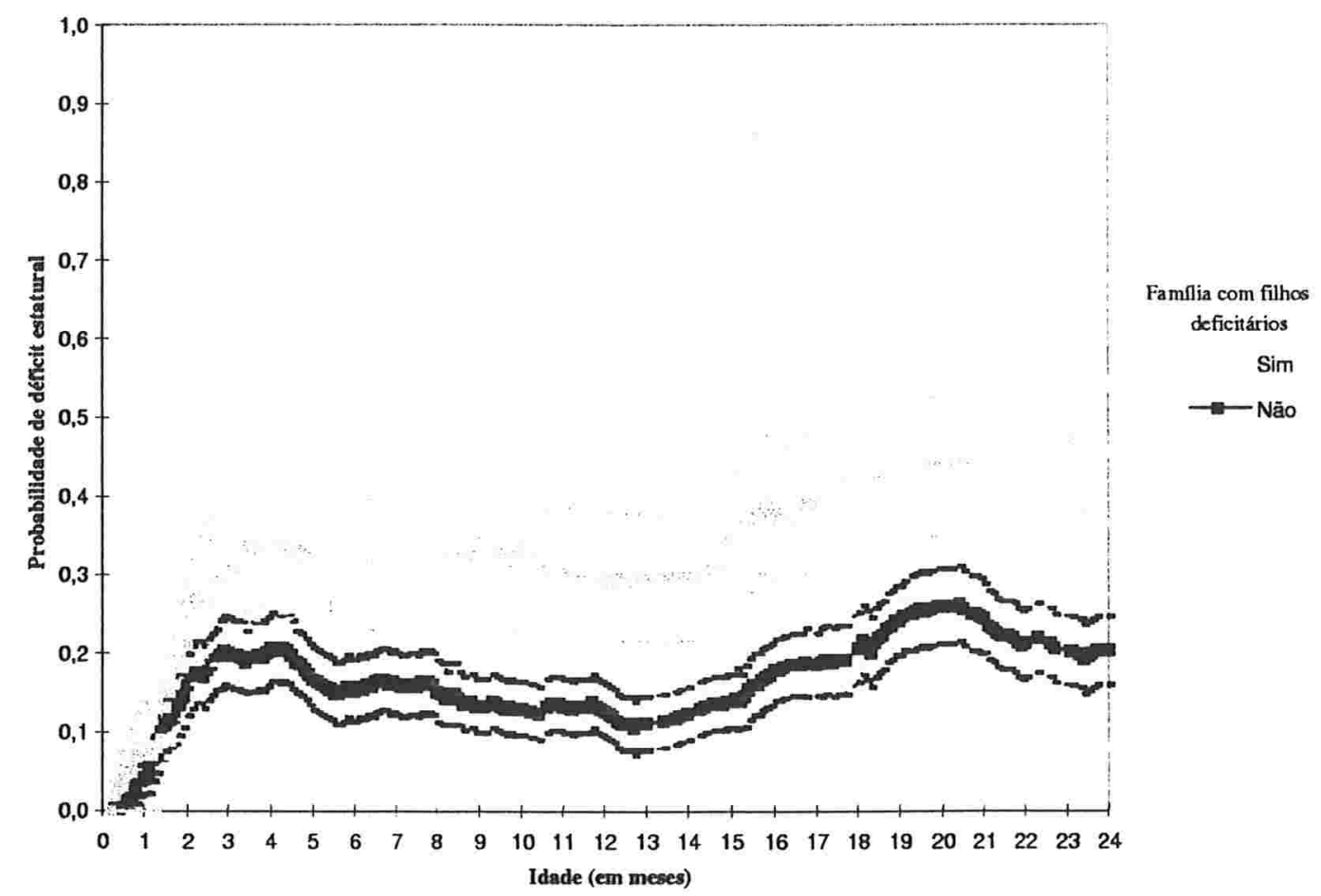

Gráfico 4.2: Probabilidade de déficit estatural segundo condição da família

Analisando o gráfico observamos que nos primeiros meses as probabilidades são, aparentemente, semelhantes nos dois grupos. A partir dos 4 meses de idade, a probabilidade de déficit estatural é visualmente maior nas crianças nascidas em famílias com outros filhos deficitários. 
$\mathrm{Na}$ maioria das idades a probabilidade de déficit em famílias deficitárias chega a ser superior ao dobro da probabilidade em famílias não deficitárias. Por exemplo, aos 8 meses as probabilidades para os grupos com e sem filhos com déficit são respectivamente 0,32 e 0,15 e aos 22 meses são 0,46 e 0,21 . Estes e outros resultados são comentados sob o ponto de vista nutricional por Lei, Chaves, Paes, Escuder, Ribeiro, Freire \& Lerner (1999).

\subsection{Inclusão de covariáveis}

Com o objetivo de considerar os efeitos de outras variáveis que poderiam estar relacionadas com as probabilidades de transição, ajustamos modelos de regressão segundo as três modelagens descritas no Capítulo 3. Foram incluídas nos modelos as seguintes variáveis:

\begin{tabular}{ll} 
Variável & Descrição \\
\hline$Z_{1}:$ Peso & Peso ao nascer (em gramas x 100) \\
$Z_{2}:$ Sexo & Sexo (1-masculino; 0-feminino) \\
$Z_{3}:$ Altmae & Altura da mãe (em cm) \\
$Z_{4}:$ Nirm1 & $N^{\circ}$ de irmãos menores de 7 anos (1-dois; 0-c.c.) ${ }^{(*)}$ \\
$Z_{5}:$ Nirm2 & $N^{0}$ de irmãos menores de 7 anos (1-três ou mais; 0-c.c.) ${ }^{(*)}$ \\
$Z_{6}:$ Família & Família com outros filhos deficitários (1-sim; 0-não) \\
$Z_{7}:$ Área & Área geográfica de moradia (1-periférica; 0-central)
\end{tabular}

$\left(^{*}\right)$ todos tinham pelo menos 1 irmão

Neste problema, os modelos de regressão foram ajustados para cada tipo de transição: transição $0 \rightarrow 1$ que representa a probabilidade de déficit estatural e transição $1 \rightarrow 0$ que corresponde à probabilidade de recuperação. Como comentamos no capítulo anterior, isto pode ser feito de uma só vez, considerando um único vetor de parâmetros $\beta$ e escrevendo convenientemente o vetor de covariáveis.

No modelo de incrementos independentes, a partir do vetor de covariáveis originais $\mathrm{Z}=$ $\left(Z_{1}, Z_{2}, Z_{3}, Z_{4}, Z_{5}, Z_{6}, Z_{7}\right)$ foram criadas as covariáveis específicas $\mathbf{Z}_{01}$ e $\mathbf{Z}_{10}$ de acordo com o tipo de transição. Assim, para cada observação $i$ o vetor de covariáveis era dado por $\mathbf{Z}_{01 i}^{T}=$ $\left(\mathbf{Z}_{i}, 0,0,0,0,0,0,0\right)$ se a transição fosse do tipo $0 \rightarrow 1$ e por $\mathbf{Z}_{10 i}^{T}=\left(0,0,0,0,0,0,0, \mathbf{Z}_{i}\right)$ se 
a transição fosse do tipo $1 \rightarrow 0$.

Nos modelos condicional e marginal, além do tipo de transição, as covariáveis foram especificadas também pela ordem em que as transições estavam ocorrendo. Embora algumas crianças tivessem feito mais de 3 transições, em ambos os modelos consideramos somente até a terceira transição e assim foram excluídas 10 observações. Neste caso, havia 42 parâmetros a serem estimados ( 7 covariáveis, 2 transições ocorrendo até 3 vezes) e portanto, no lugar de apenas dois vetores $14 \times 1$ como no modelo AG, as covariáveis específicas eram dadas por seis vetores $\mathbf{Z}_{011}, \mathbf{Z}_{012}, \mathbf{Z}_{013}, \mathbf{Z}_{101}, \mathbf{Z}_{102}$ e $\mathbf{Z}_{103}$, cada um com dimensão $42 \times 1$. Os resultados correspondentes aos modelos diferenciados pelo número de transições são apresentados no Apêndice C.

É interessante observar que as estimativas referentes ao primeiro estrato (primeira vez em que a transição ocorre) são exatamente as mesmas nos dois modelos. Isto acontece porque todas as observações são consideradas em risco para a primeira transição e portanto o conjunto de risco é o mesmo. A partir da segunda transição, os conjuntos de risco para o modelo marginal são bem maiores que no modelo condicional que considera somente os que já fizeram a transição uma vez e com isso os resultados são completamente diferentes.

Para tornar possível a comparação entre os modelos, fizemos algumas suposições em relação ao número de transições. Assumimos que o número de vezes que a criança esteve deficitária não influencia a probabilidade de tornar-se deficitária novamente, assim como o número de vezes que ela consegue se recuperar também não aumenta a chance de uma nova recuperação.

Assim, no modelo condicional ajustamos um modelo estratificado segundo o número de transições, porém estimando um único vetor de parâmetros através da especificação das covariáveis, segundo apenas o tipo de transição, ou seja, $\mathbf{Z}_{01}$ e $\mathbf{Z}_{10}$. No modelo marginal, calculamos os parâmetros combinando as estimativas dos três modelos marginais considerando uma combinação linear conforme sugestão descrita no Capítulo 3. 


\section{Resultados dos modelos de regressão}

As Tabelas 4.1.e 4.2 apresentam as estimativas produzidas pelos três modelos. Nos modelos referentes à transição $0 \rightarrow 1$, os resultados dos três métodos apontam para 0 mesmo sentido. As variáveis consideradas significativas para este tipo de transição foram o peso ao nascer, a altura da mãe, o número de irmãos e a condição da família. Analisando

os riscos $(\exp (\hat{\beta}))$ concluímos que quanto maior o peso e maior a estatura da mãe, menor o risco de déficit estatural. Concluímos também que o risco é maior para crianças com mais de dois irmãos menores de 7 anos e crianças nascidas em famílias que já tem outros filhos deficitários.

$\mathrm{Na}$ análise da transição $1 \rightarrow 0$, o peso e altura da mãe não aparecem como significativas, ou seja, apesar de terem sido consideradas importantes para o risco de déficit estatural, essas variáveis não influenciam na chance de recuperação. Por outro lado, a área de moradia aparece como um fator importante, indicando que crianças deficitárias que moram na periferia têm menos chance de se recuperar do que crianças que moram em uma região mais central.

A condição da família é a única variável cujos resultados diferem entre os modelos. $\mathrm{O}$ modelo de incrementos independentes e o modelo condicional indicam que o fato da família já ter outros filhos com déficit dificulta significativamente a recuperação $(p<0,01)$. No modelo marginal, embora o risco seja semelhante aos outros modelos $(0,74)$ o teste para avaliar a significância do parâmetro produziu um nível descritivo menos significativo $(0,08)$.

De uma maneira geral, os três modelos produzem resultados semelhantes. As estimativas dos riscos são muito próximas, exceto para o número de irmãos e condição da família cujos riscos para o déficit estatural são consideravelmente maiores no modelo marginal. Convém observar também que as estimativas modelo marginal tem sempre a maior variabilidade justamente por serem resultados de combinações entre 3 modelos. 

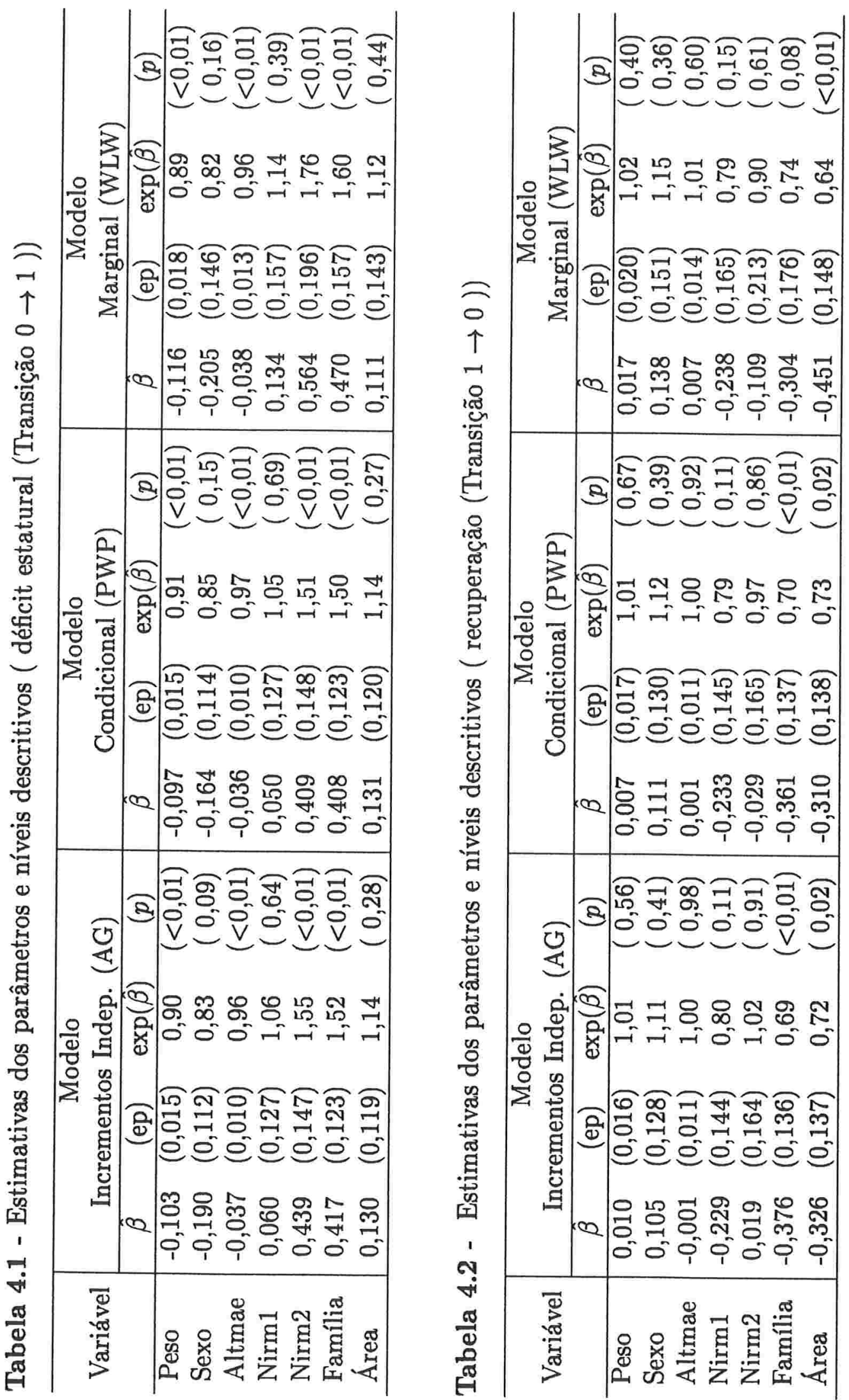
A semelhança entre os resultados dos três modelos pode estar ocorrendo provavelmente porque, neste problema, o número de transições anteriores talvez não seja tão importante. Para verificar essa suposição com mais cuidado ajustamos um novo modelo de incrementos independentes incluindo a informação sobre o número de transições anteriores. Para isso criamos, para cada tipo de transição, duas novas variáveis:

$$
\begin{aligned}
& \operatorname{Ant} 1(\mathrm{t})= \begin{cases}1 & \text { se a transição ocorreu } 1 \text { vez até o instante } t \\
0 & \text { caso contrário } ;\end{cases} \\
& \operatorname{Ant2}(\mathrm{t})= \begin{cases}1 & \text { se a transição ocorreu } 2 \text { vezes ou mais até o } \\
& \text { instante } t ; \\
0 & \text { caso contrário }\end{cases}
\end{aligned}
$$

Os resultados desse novo modelo, apresentados na Tabela 4.3, indicam que tanto para a transição do tipo $0 \rightarrow 1$ quanto para a transição $1 \rightarrow 0$ o número de transições anteriores não é significativo. Isto sugere que de fato, a ocorrência das transições pouco altera as intensidades, o que pode explicar a semelhança entre os modelos AG e PWP, uma vez que a única diferença entre eles é a especificação das intensidades padrão segundo o número de transições.

Tabela 4.3 - Estimativas dos parâmetros e níveis descritivos ( modelo AG incluindo número de transições anteriores )

\begin{tabular}{l|cccc|cccc}
\hline \multirow{2}{*}{ Variável } & \multicolumn{6}{|c}{ Modelo de Incrementos Independentes (AG) } \\
\cline { 2 - 9 } & $\hat{\beta}$ & $(\mathrm{ep})$ & $\exp (\hat{\beta})$ & $(p)$ & $\hat{\beta}$ & $(\mathrm{ep})$ & $\exp (\hat{\beta})$ & $(p)$ \\
\hline Peso & $-0,097$ & $(0,015)$ & 0,91 & $(<0,01)$ & 0,007 & $(0,016)$ & 1,01 & $(0,67)$ \\
Sexo & $-0,168$ & $(0,113)$ & 0,85 & $(0,14)$ & 0,094 & $(0,129)$ & 1,10 & $(0,47)$ \\
Altmae & $-0,035$ & $(0,010)$ & 0,97 & $(<0,01)$ & $-0,001$ & $(0,011)$ & 1,00 & $(0,92)$ \\
Nirm1 & 0,059 & $(0,127)$ & 1,06 & $(0,64)$ & $-0,240$ & $(0,144)$ & 0,79 & $(0,10)$ \\
Nirm2 & 0,414 & $(0,148)$ & 1,51 & $(<0,01)$ & 0,007 & $(0,165)$ & 1,01 & $(0,97)$ \\
Família & 0,396 & $(0,123)$ & 1,49 & $(<0,01)$ & $-0,371$ & $(0,137)$ & 0,69 & $(0,01)$ \\
Área & 0,123 & $(0,119)$ & 1,13 & $(0,30)$ & $-0,318$ & $(0,137)$ & 0,73 & $(0,02)$ \\
Ant1 & 0,177 & $(0,143)$ & 1,19 & $(0,22)$ & $-0,191$ & $(0,158)$ & 0,83 & $(0,23)$ \\
Ant2 & 0,336 & $(0,237)$ & 1,40 & $(0,16)$ & 0,006 & $(0,265)$ & 1,01 & $(0,98)$ \\
\hline
\end{tabular}




\section{Probabilidades de transição}

Para comparar os modelos graficamente calculamos as probabilidades de transição em função da idade através da expressão (3.10) considerando uma observação hipotética. Os Gráficos 4.3 e 4.4 apresentados a seguir correspondem às probabilidades calculadas para uma criança com as seguintes características:

Peso ao nascer: $3200 \mathrm{Kg}$

Sexo: masculino

Altura da mãe: $1,60 \mathrm{~cm}$

Número de irmãos: 4

Condição da família: com filhos deficitários

Área de moradia: periférica

Os gráficos indicam o que já foi observado na análise das estimativas, ou seja, os resultados são bastante próximos, principalmente entre os modelos AG e PWP. Embora o modelo marginal se diferencie levemente nos gráficos, essas diferenças não chegam a mais de $5 \%$. A maior diferença observada foi aos 20 meses onde a probabilidade de déficit sob o modelo marginal foi de 0,54 contra 0,49 dos outros dois modelos.

Com o objetivo de visualizar a influência da condição da família considerando desta vez o modelo de regressão, consideramos uma nova criança com as mesmas características da primeira só que nascida em uma família sem filhos deficitários. O Gráfico 4.5 mostra as probabilidades de déficit estatural calculadas pelo modelo AG para as duas crianças.

É interessante notar que, mesmo não sendo exatamente proporcionais, as probabilidades acompanham a proporcionalidade suposta no modelo de Cox. Isto acontece porque os estimadores de Nelson-Aalen calculados em cada instante $\Delta \hat{\mathbf{A}}\left(T_{i}\right)$ utilizados para o cálculo dos estimadores $\hat{\mathbf{P}}\left(T_{i}\right)$ são proporcionais. Assim, podemos observar que a partir dos 2 meses de idade até o fim do período de observação, as diferenças permanecem aproximadamente constantes ficando, em média, em torno de $15 \%$. 


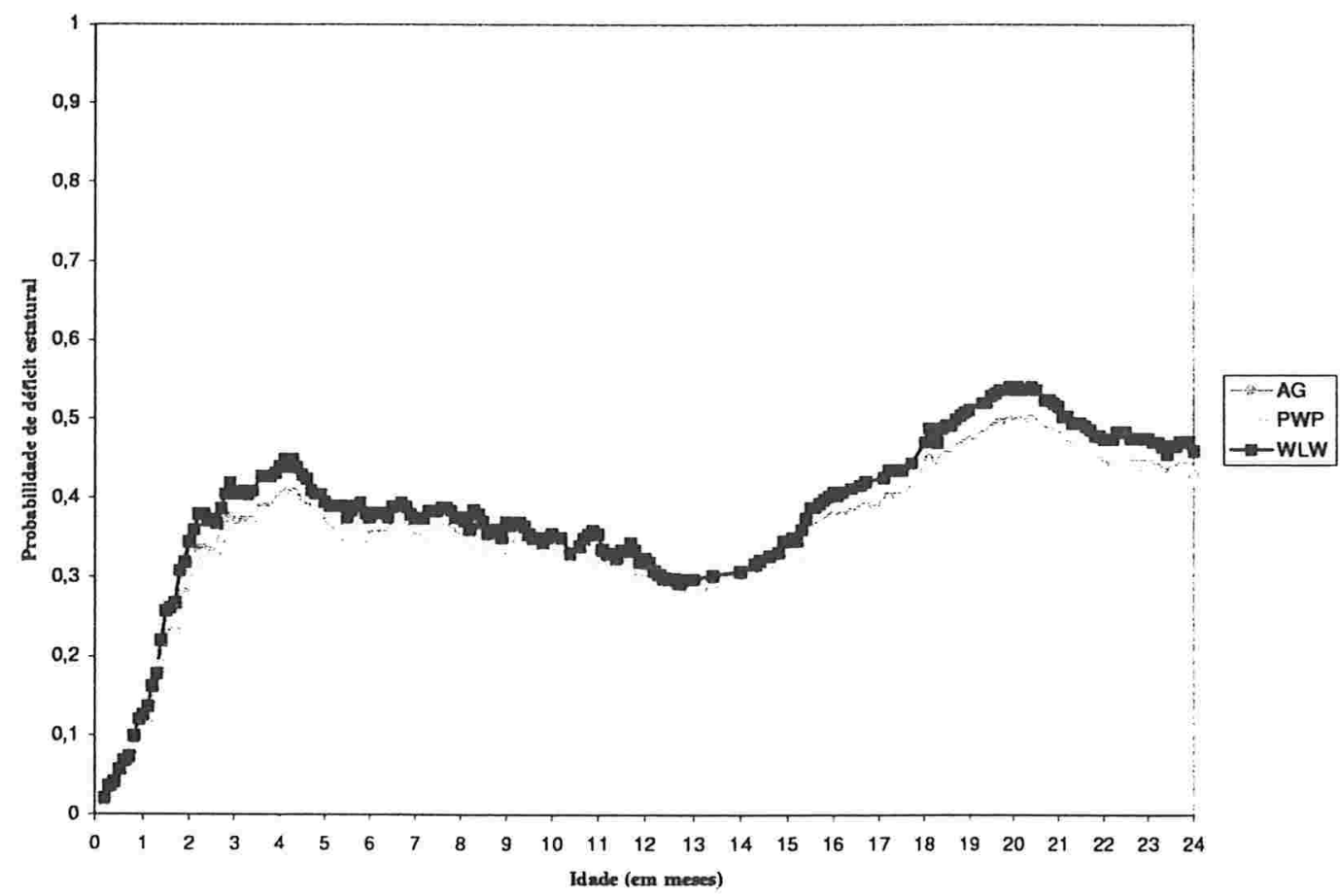

Gráfico 4.3: Probabilidade de déficit estatural - comparação entre modelos

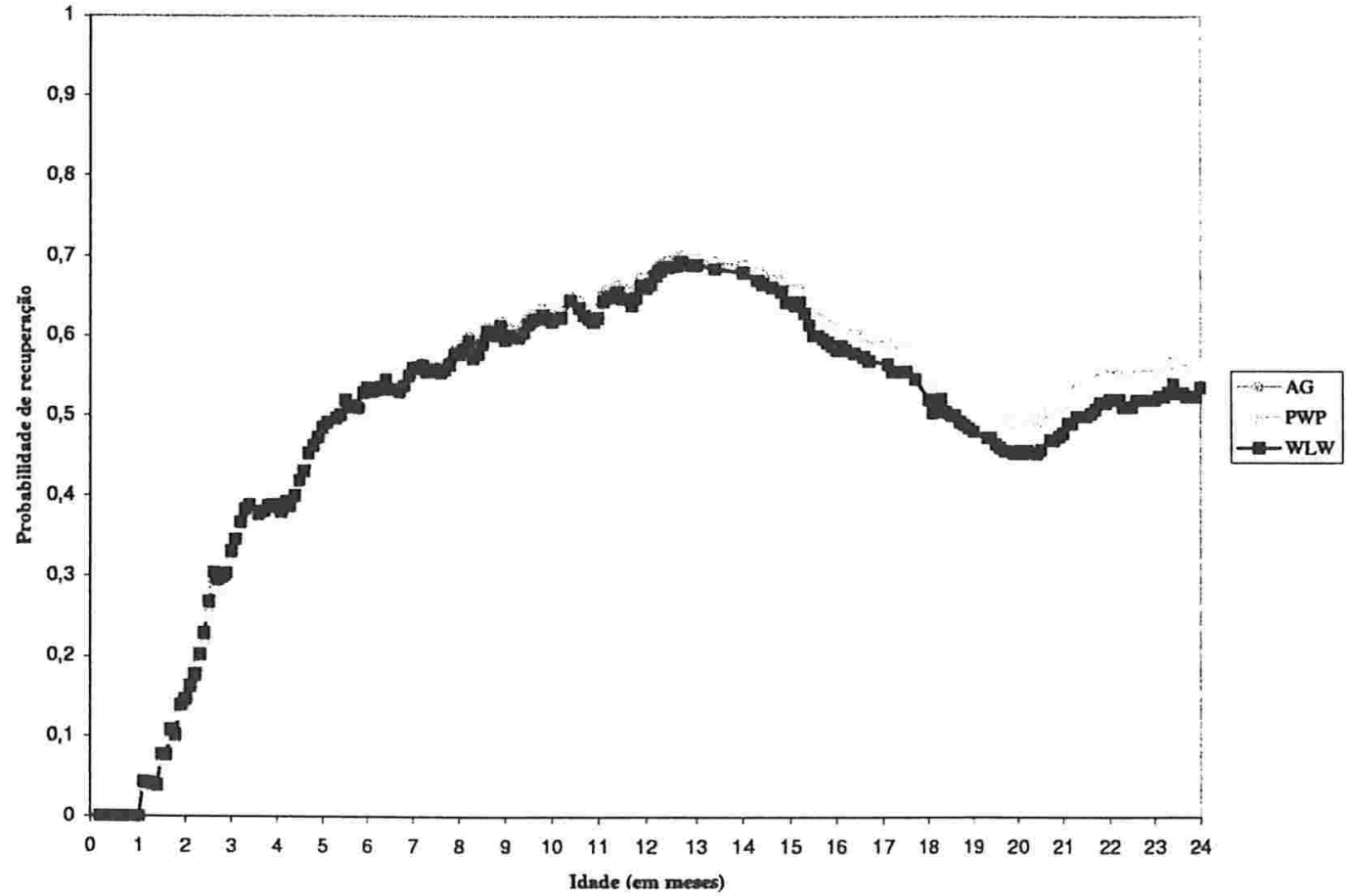

Gráfico 4.4: Probabilidade de recuperação - comparação entre modelos 


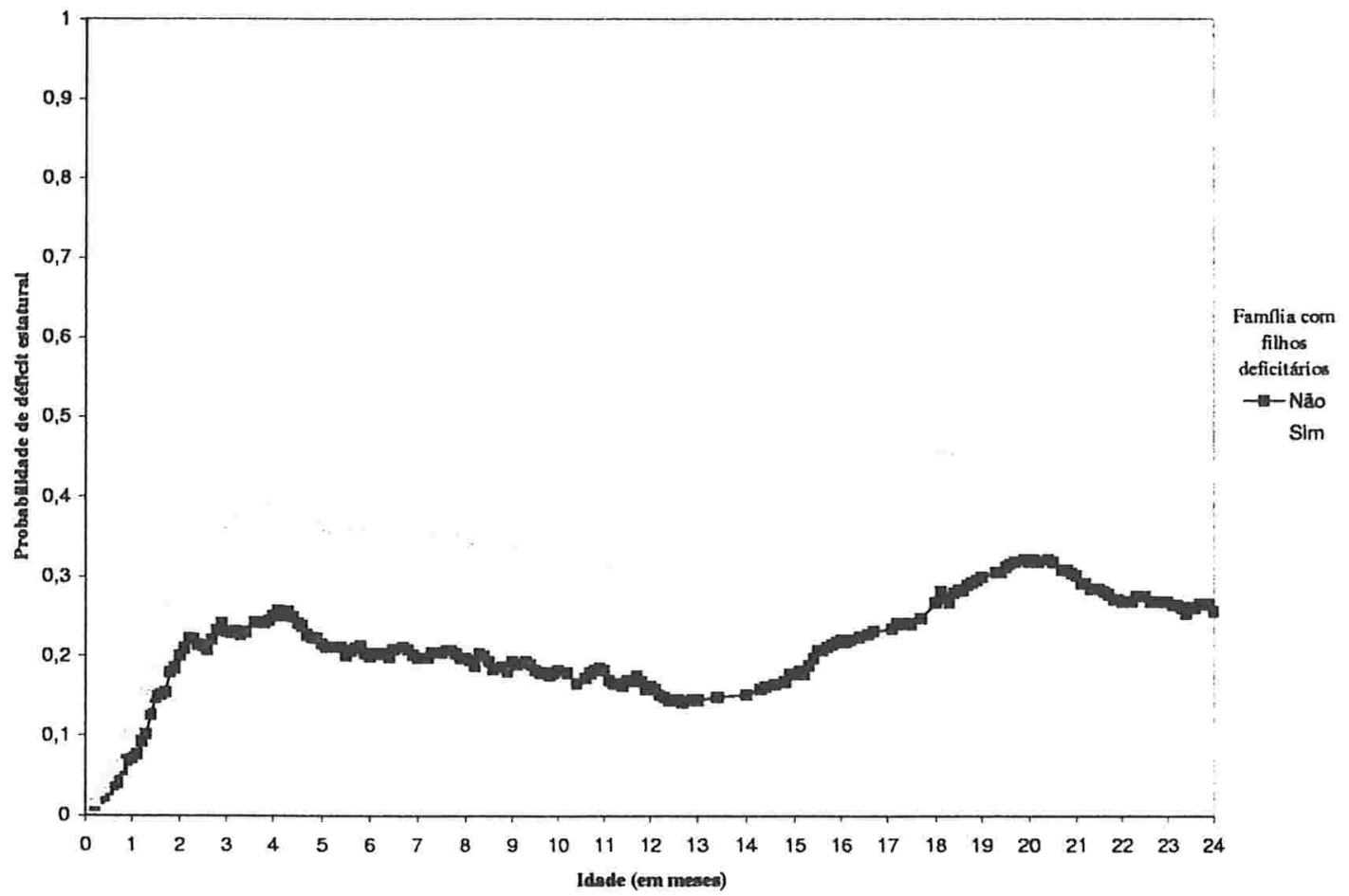

Gráfico 4.5: Probabilidade de déficit estatural - comparação segundo a condição da família 
Com o intuito de analisar a intensidade com que algumas variáveis interferiam no cálculo das probabilidades, comparamos duas crianças cujas características diferiam em relação as variáveis identificadas como significativas nos modelos de regressão. Assim, consideramos duas crianças, uma com as condições favoráveis e outra com as condições desfavoráveis relativamente a essas variáveis. As características de cada criança e o gráfico com as probabilidades de déficit são apresentados a seguir.

\begin{tabular}{lcc} 
Variável & Criança 1 & Criança 2 \\
\hline Peso & $3 \mathrm{Kg}$ & $3,5 \mathrm{Kg}$ \\
Sexo & Feminino & Feminino \\
Altura da mãe & $1,52 \mathrm{~m}$ & $1,62 \mathrm{~m}$ \\
Número de irmãos & 4 & 1 \\
Família com filhos deficitários & Sim & Não \\
Área & Central & Central \\
\hline
\end{tabular}

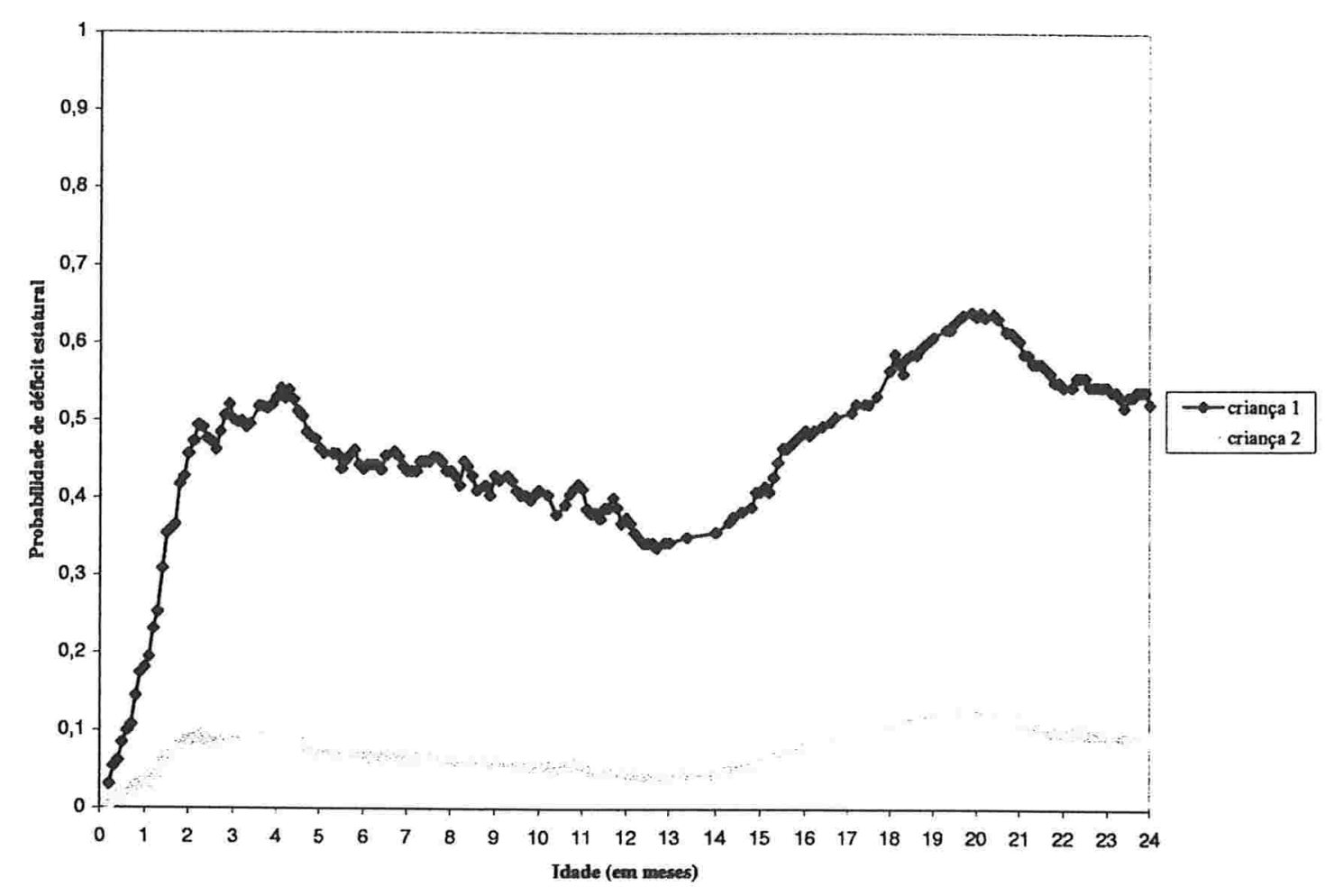

Gráfico 4.6: Probabilidade de défict estatural - comparação segundo variáveis significativas nos modelos de regressão

Analisando o gráfico observamos que o menor peso e altura, um número maior de irmãos e a presença de irmãos deficitários agravam em muito a condição da criança, aumentando significativamente o risco de déficit estatural. 


\subsection{Considerações finais}

A escolha do modelo deve depender principalmente do tipo de dado e das hipóteses que se pretende verificar. Isto envolve entre outras coisas a estrutura dos conjuntos de risco e a escala de tempo a ser considerada. Lin (1994) sugere que para eventos do mesmo tipo se utilize o modelo AG que é o mais simples, principalmente quando houver poucas recorrências (poucos indivíduos com dois eventos ou mais). No caso de haver um interesse nos tempos entre as ocorrências dos eventos, podemos considerar o modelo PWP apresentado em (3.7).

No exemplo analisado, os três modelos produziram resultados muito semelhantes e a utilização de um ou outro pouco altera as conclusões do estudo. Isto pode estar acontecendo porque a história anterior praticamente não modifica as intensidades de transição, isto é, o número de eventos anteriores de uma mesma criança não aumenta ou diminui o risco de um novo evento.

Neste caso, o modelo de incrementos independentes é suficiente para responder de modo satisfatório as hipóteses da análise, além da facilidade de processamento. No caso do número de transições ser importante, acreditamos que as informações correspondentes possam ser bem acomodadas neste mesmo modelo sob a forma de covariáveis. Os modelos especificados desta forma, além de exigirem um número menor de parâmetros, facilitam sua interpretação em termos práticos.

Quanto ao modelo marginal, sugerimos que seja utilizado quando de fato houver suspeitas sobre as especificações do modelo de Cox. Isto porque do ponto de vista operacional, este modelo é muito mais trabalhoso e requer alguma programação ainda não implementada pelos pacotes computacionais convencionais (ver Apêndice B). 


\section{Apêndice A}

\section{Teoremas}

Teorema A.1 (Teorema II.6.2 em Andersen et al. (1993))

Seja $\mathbf{Y}=\pi(\mathbf{I}+d \mathbf{X}) e \mathbf{Y}^{\prime}=\pi\left(\mathbf{I}+d \mathbf{X}^{\prime}\right)$. Então,

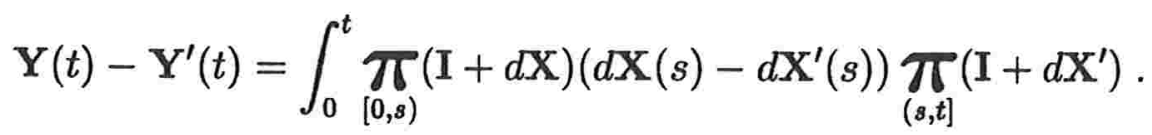

Quando $\mathbf{Y}^{\prime}(t)$ é não singular,

$$
\mathbf{Y}(t) \mathbf{Y}^{\prime}(t)^{-1}-\mathbf{I}=\int_{0}^{t} \mathbf{Y}\left(s^{-}\right)\left(d \mathbf{X}(s)-d \mathbf{X}^{\prime}(s)\right)\left(\mathbf{Y}^{\prime}(s)\right)^{-1}
$$

Teorema A.2 Seja $N(t)$ um processo de contagem e $\Lambda(t)$ seu compensador e seja $H(t)$ um processo previsível e limitado. A integral estocástica

$$
L(t)=\int_{0}^{t} H(u) d\{N(u)-\Lambda(u)\}
$$

é um martingal de média 0 . 
Teorema A.3 (Versão simplificada do Teorema II.6.7 enunciado por Andersen et al. (1993)) Seja um processo de Markov com espaço de estados $\{1,2, \ldots, k\}$ e seja A sua medida de intensidade. Então a matriz de transição do processo é

$$
\mathbf{P}(s, t)=\underset{(s, t]}{\pi}(\mathbf{I}+d \mathbf{A}), \quad s \leq t .
$$

Proposição 1:(versão simplificada da Proposição II.4.1 (Andersen et al. 1993)) Seja o processo de contagem $\mathrm{N}(\mathrm{t})$ com intensidade $\lambda$. Seja $M=N-\int \lambda$ e seja $\mathbf{H}$ limitado e previsível. Então $\mathbf{M}$ e $\int \mathbf{H} d \mathbf{M}$ são martingais com:

$$
\begin{aligned}
& \langle\mathbf{M}\rangle=\operatorname{diag} \int \boldsymbol{\lambda}, \\
& \left\langle\int \mathbf{H d} \mathbf{M}\right\rangle=\int \mathbf{H}(\operatorname{diag} \boldsymbol{\lambda}) \mathbf{H}^{T} .
\end{aligned}
$$

Considerando $\delta_{h l}$ o delta de Kronecker,

$$
\begin{aligned}
& \left\langle M_{h}, M_{l}\right\rangle=\delta_{h l} \int \lambda_{h}, \\
& \left\langle\sum_{h} \int H_{j h} d M_{h}, \sum_{l} \int H_{j l l} d M_{l}\right\rangle=\sum_{h} \int H_{j h} H_{j l h} \lambda_{h} .
\end{aligned}
$$

Proposição 2: Na Proposição 1, se $\mathbf{N}, \Lambda$ e $\mathbf{H}$ são processos univariados, então

$$
\left\langle\int \mathbf{H} d \mathbf{M}\right\rangle=\int \mathbf{H}^{2} \boldsymbol{\lambda} .
$$

Proposição 3: Sejam $\mathbf{H}=\left\{H_{h j}\right\}$ matriz $p \times q$ e $\mathbf{K}=\left\{K_{h j}\right\}$ matriz $r \times s$. Se $\mathbf{H}$ e $\mathbf{K}$ são processos previsíveis e $M$ é um martingal, então

$$
\left\langle\int \mathbf{H} d \mathbf{M K}\right\rangle=\int\left(\mathbf{K}^{T} \otimes \mathbf{H}\right) d\langle\mathbf{M}\rangle\left(\mathbf{K} \otimes \mathbf{H}^{T}\right)
$$




\section{Apêndice B}

\section{Aspectos computacionais}

A principal dificuldade da parte computacional talvez seja a manipulação dos bancos de dados. Na maioria dos arquivos, cada linha corresponde a um indivíduo e todas as informações sobre ele são dispostas nas colunas. No caso de eventos recorrentes cada indivíduo contribui com várias observações, tantas forem as recorrências do evento para aquele indivíduo. Como para a análise cada observação deve corresponder a um evento, as observações de um mesmo indivíduo devem estar dispostas em diversas linhas.

Mediante alguma programação, é possível a partir do banco original modificar o formato dos dados de forma conveniente para que as técnicas possam ser aplicadas. Entretanto, quando não há um domínio nesse campo, transformar os dados manualmente pode ser um processo bastante trabalhoso.

No exemplo apresentado no Capítulo 4, após o cálculo das idades e da criação das variáveis que definem o déficit estatural, os dados medidos ao longo do tempo tinham a seguinte forma:

\begin{tabular}{cccccccccc} 
criança & idade $_{1}$ & def $_{1}$ & idade $_{2}$ & def $_{2}$ & $\ldots$ & idade $_{16}$ & def $_{16}$ & idade $_{17}$ & def $_{17}$ \\
\hline 1 & 0,2 & 0 & 1,3 & 1 & $\ldots$ & 23,9 & 1 & 25,1 & 0 \\
2 & 0,3 & 0 & 1,5 & 0 & $\ldots$ & 24,2 & 1 &. &. \\
$\vdots$ & $\vdots$ & $\vdots$ & $\vdots$ & $\vdots$ & $\vdots$ & $\vdots$ & $\vdots$ & $\vdots$ & $\vdots$ \\
\hline
\end{tabular}

onde $i$ dade $_{j}$ é a idade da criança (em meses) na $j$-ésima visita, $j=1, \ldots, 17$ e de $f_{j}$ é o estado da criança na $j$-ésima visita (1- com déficit estatural , 0 - sem déficit estatural). 


\section{Transições}

Consideramos concretizada cada transição quando o estado da criança em uma determinada visita era diferente do estado na visita anterior. Desta forma, para cada visita $j$ criamos uma variável indicadora de transição, isto é,

$$
\operatorname{transic}_{j}=\left\{\begin{array}{l}
0 \text { se não ocorreu transição } ; \\
1 \text { se ocorreu a transição } 0 \rightarrow 1 ; \\
2 \text { se ocorreu a transição } 1 \rightarrow 0 .
\end{array}\right.
$$

A partir dessa nova variável, consideramos somente as idades em que foram observadas transições $\left(\right.$ transic $_{j}=1$ ou transic $_{j}=2$ ). As idades em que não havia transição $\left(\right.$ transic $_{j}=0$ ) só eram aproveitadas quando correspondiam à última observação (visita) da criança sendo portanto considerada uma censura.

\section{Censuras}

Se na última visita não havia sido observada transição essa observação era dada como censurada. A censura era considerada do tipo 1 ou 2 conforme o estado associado à última visita. Por exemplo, uma criança que estava no estado 0 (sem déficit) e não teve uma transição nesta última visita era considerada como uma observação censurada para a transição do tipo 0-1. Se a criança ficasse deficitária e continuasse assim até a última visita, era considerada como observação censurada para a transição 1-0.

\section{Estimação não paramétrica}

O cálculo dos estimadores não paramétricos são simples e podem ser feitos inclusive em planilhas eletrônicas como o Excel. Neste caso, é necessário ordenar segundo os tempos de transição $T_{i}$ e criar as colunas correspondentes a $N_{01}\left(T_{i}\right)$ e $N_{10}\left(T_{i}\right), Y_{0}\left(T_{i}\right)$ e $Y_{1}\left(T_{i}\right)$. Estes cálculos também podem ser facilmente efetuados em programas estatísticos usuais. No SAS por exemplo, o produto das matrizes pode ser feito diretamente através do proc IML enquanto que no Excel isso é feito elemento a elemento. 


\section{Modelos de regressão}

Para os modelos de regressão também podem ser usados os programas estatísticos usuais. A versão 6.12 do SAS (proc PHREG) já considera dados na forma de processos de contagem, em que cada indivíduo entra como várias observações. Neste caso, a informação sobre os tempos é dada através de duas variáveis (tempo1 e tempo2) que representam o intervalo no qual o indivíduo permanece em risco.

A diferença entre os três modelos está principalmente na disposição do banco de dados. As entradas de dados para os modelos de incrementos independentes e condicional são semelhantes. A principal diferença é que no segundo deve-se considerar uma variável adicional que indica a ordem em que a transição está ocorrendo. Além disso, para se obter um modelo estratificado, eventualmente pode ser necessário excluir algumas observações caso os últimos estratos tenham pouca freqüência.

Já no modelo marginal, a entrada dos dados é bem mais complexa devido à descontinuidade dos períodos de observação. Como estamos observando transições, para cada tipo de transição os períodos de observação nunca são contínuos. Isto acontece porque a partir do momento em que o indivíduo é submetido a um tipo de transição ele sai do conjunto de risco e só volta a ser considerado quando retornar ao mesmo estado. Como cada observação é tratada como um vetor de tempos, cada indivíduo contribui com várias observações censuradas.

Essas diferenças podem ser melhor entendidas através de um exemplo. Considere a criança $\mathrm{n}^{\circ} 24$ que ficou deficitária aos 14,3 meses, conseguiu recuperar-se aos 17,4 meses, ficou deficitária novamente aos 20,4 meses e permaneceu com déficit até sua última visita quando tinha 24,6 meses. Nos modelos AG e PWP esta criança entra com as seguintes observações:

\begin{tabular}{cccccc}
\hline ind & regist* & transic & tempo1 & tempo2 & evento \\
\hline 24 & 1 & 1 & 0 & 14,3 & 1 \\
24 & 1 & 2 & 14,3 & 17,4 & 1 \\
24 & 2 & 1 & 17,4 & 20,4 & 1 \\
24 & 2 & 2 & 20,4 & 24,6 & 0 \\
\hline
\end{tabular}

\footnotetext{
* ordem da transição (só para o modelo PWP)
} 
No modelo marginal, cada observação conta como um vetor de tempos. 'Para a transição do tipo $0 \rightarrow 1$, essa criança tem dois períodos de observação: 0 a 14,3 meses e 17,4 a 20,4 meses. No primeiro período, em que ocorreu a primeira transição, a observação é contada como censura para a segunda e terceira transições. No segundo período, onde foi observada a segunda transição, a observação é considerada como censura para a terceira. Para a transição $1 \rightarrow 0$ os períodos de observação são 14,3 a 17,4 meses e 20,4 a 24,6 meses. Como a transição só foi observada uma vez, em ambos os períodos consideramos censuras para a segunda e terceira transições. Desta forma, as observações referentes à essa criança são dispostas como:

\begin{tabular}{cccccc}
\hline ind & regist & tipotran & tempo1 & tempo2 & evento \\
\hline 24 & 1 & 1 & 0 & 14,3 & 1 \\
24 & 2 & 1 & 0 & 14,3 & 0 \\
24 & 3 & 1 & 0 & 14,3 & 0 \\
24 & 2 & 1 & 17,4 & 20,4 & 1 \\
24 & 3 & 1 & 17,4 & 20,4 & 0 \\
24 & 1 & 2 & 14,3 & 17,4 & 1 \\
24 & 2 & 2 & 14,3 & 17,4 & 0 \\
24 & 3 & 2 & 14,3 & 17,4 & 0 \\
24 & 2 & 2 & 20,4 & 24,6 & 0 \\
24 & 3 & 2 & 20,4 & 24,6 & 0 \\
\hline
\end{tabular}

Assim, as informações de 345 crianças foram transformadas em 922 registros para o ajuste dos modelos AG e PWP e em 2271 registros para o modelo marginal.

\section{Covariáveis}

Como os dados de um mesmo indivíduo estão em várias linhas, os valores das covariáveis devem ser repetidos em todas as linhas. As covariáveis podem entrar na forma original e depois serem transformadas em covariáveis específicas. No proc DATA do SAS cada covariável específica pode ser criada diretamente por uma única operação. Por exemplo, a variável peso especificada pelo tipo de transição 0-1 no segundo estrato é obtida simplesmente pelo comando peso012=peso* $(\text { tipotran }=1)^{*}($ registro=2). Este comando cria uma nova variável (peso012) atribuindo o valor do peso para as observações que satisfazem as condições especificadas (transição $0 \rightarrow 1$ ocorrendo pela segunda vez) e zero ao resto. 


\section{Variância Robusta}

Os resíduos leverage utilizados para o cálculo da matriz de covariância robusta do modelo marginal correspondem aos resíduos DFBETA obtidos pelo SAS. Porém, como no banco de dados cada observação corresponde a uma transição, os resíduos obtidos correspondem à influência de cada observação e não de cada indivíduo. Para obter os resíduos por indivíduo é preciso reordenar o arquivo por tipo de transição e registro e somar os resíduos dentro de cada indivíduo. No SAS, isto pode ser feito pelo proc MEANS.

Therneau \& Hamilton (1997) mostram que a variância robusta também pode ser facilmente obtida através do S-Plus (versão 3.3). Outra alternativa é utilizar o MULCOX2, um programa em FORTRAN desenvolvido por Lin (1993) para implementar o método de Wei et al. (1989). A entrada do banco de dados é a mesma e o programa fornece diretamente, entre outras coisas, as estimativas das variâncias usuais e robustas de cada parâmetro e as estatísticas de Wald para testar o efeito dos parâmetros. Os modelos AG e PWP também podem ser ajustados através do MULCOX2, modificando convenientemente o formato da entrada de dados. O programa permite ainda, a partir da construção adequada dos contrastes, testar outras hipóteses de interesse.

O teste de Wald utilizando a variância robusta, a estimação do parâmetro comum e o teste para avaliar a significância desse novo parâmetro ainda não foram implementados no SAS, porém podem ser calculados pelo proc IML.

\section{Matriz de probabilidades de transição}

Os cálculos das matrizes de probabilidades de transição, tanto para a estimação paramétrica quanto para a semiparamétrica, também podem ser feitos utilizando o proc $I M L$.

No nosso caso, a principal dificuldade foi incorporar a informação sobre os conjuntos de risco nesta formulação. Para isso foi necessário criar duas matrizes $Y_{0}$ e $Y_{1}$ com dimensões $n \times$ ntemp onde $n$ é o número de indivíduos e ntemp o número de tempos distintos em que era observada alguma transição. 


\section{Apêndice C}

\section{Tabelas complementares}




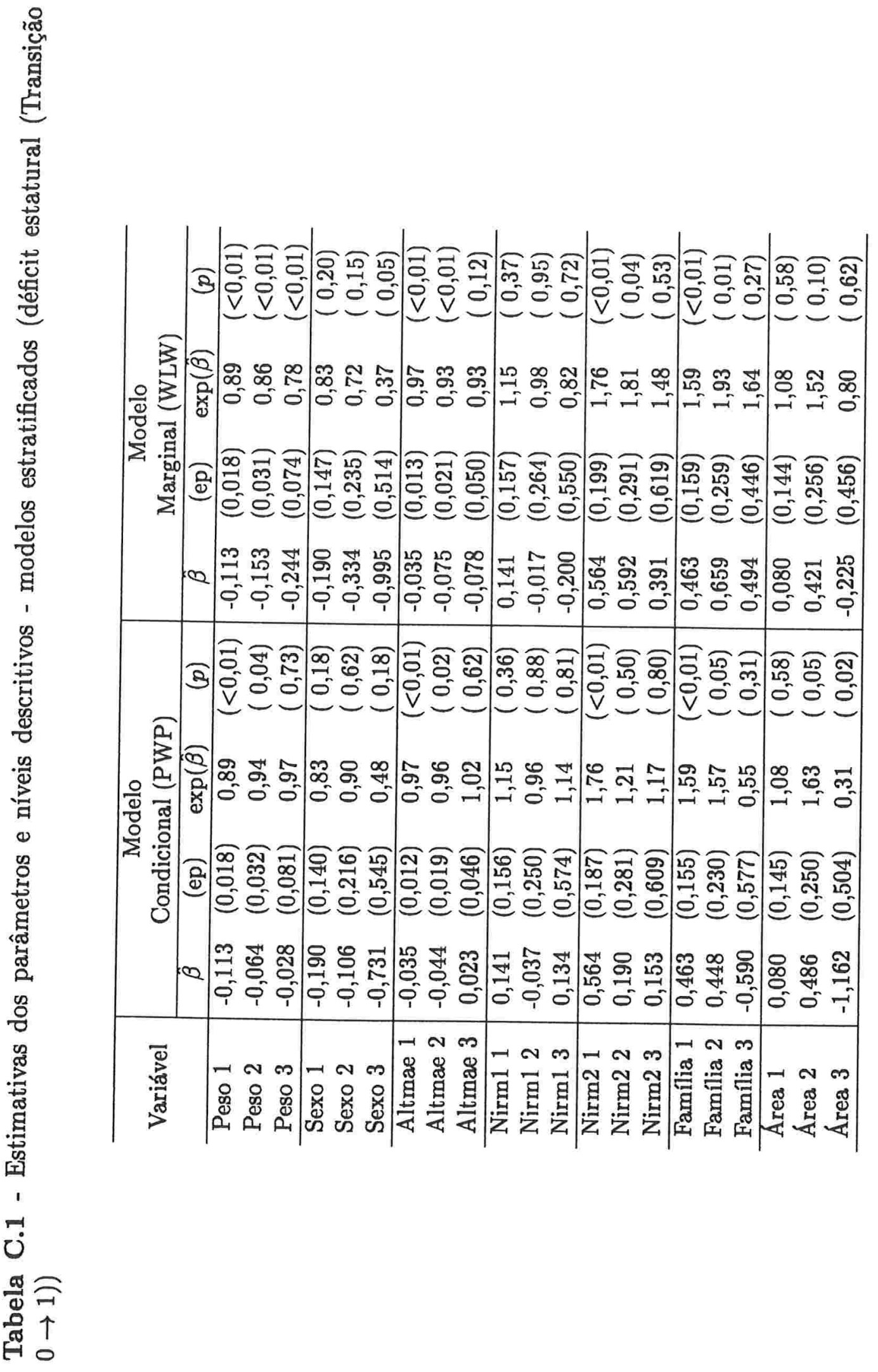




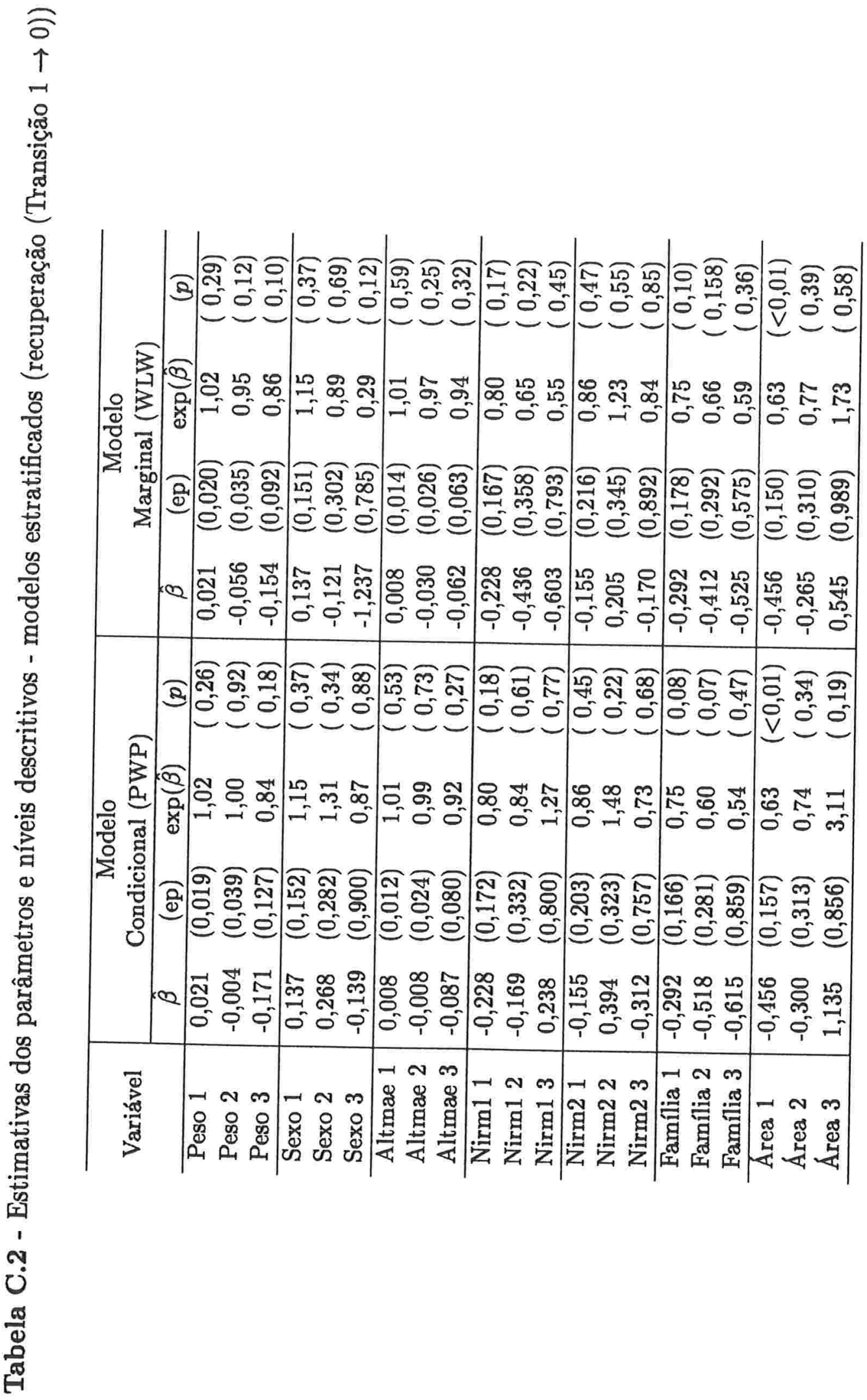




\section{Referências Bibliográficas}

Aalen, O. (1978). Nonparametric inference for a family of counting processes, The Annals of Statistics 6: 701-726.

Aalen, O. e Johansen, S. (1978). An empirical transition matrix for non-homogeneous markov chains based on censored observations, Scandinavian Journal of Statistics 5: $141-150$.

Andersen, P. e Borgan, O. (1985). Counting process models for life history data: A review, Scandinavian Journal of Statistics 12: 97-158.

Andersen, P., Borgan, O., Gill, R. e Keiding, N. (1993). Statistical Models Based on Counting Processes, Springer-Verlag, New York.

Andersen, P. e Gill, R. (1982). Cox's regression model for counting processes: A large sample study, The Annals of Statistics 10: 1100-1120.

Andersen, P., Hansen, L. e Keiding, N. (1991). Non-and semi-parametric estimation of transition probabilities from censored observation of a non-homogeneous Markov process, Scandinavian Journal of Statistics 18: 153-167.

Barai, U. e Teoh, N. (1997). Multiple statistics for multiple events, with application to repeated infections in the growth factor studies, Statistics in Medicine 16: 941-949.

Cai, J., Sen, P. e Zhou, H. (1999). A random effects model for multivariate failure time data from multicenter clinical trials, Biometrics 55: 182-189.

Cain, K. e Lange, N. (1984). Approximate case influence for the proportional hazards regression model with censored data, Biometrics 40: 493-499. 
Cook, R. (1995). The design and analysis of randomized trials with recurrent events, Statistics in Medicine 14: 2081-2098.

Cook, R. e Lawless, J. (1997a). Marginal analysis of recurrent events and a terminating event, Statistics in Medicine 16: 911-924.

Cook, R. e Lawless, J. (1997b). An overview of statistical methods for multiple failure time data in clinical trials - discussion, Statistics in Medicine 16: 841-851.

Cox, D. (1972). Regression models and life-tables (with discussion), Journal of the Royal Statistical Society, Ser. B 34: 187-220.

Cox, D. (1975). Partial likelihood, Biometrika 62: 269-276.

Doganaksoy, N. e Nelson, W. (1998). A method to compare two samples of recurrence data, Lifetime Data Analysis 4: 51-63.

Fleming e Harrington (1991). Counting Processes and Survival Analysis, Wiley, New York.

Gill, R. (1984). Understanding Cox's regression model: A martingale approach, Journal of the American Statistical Association 79: 441-447.

Gill, R. e Johansen, S. (1990). A survey of product-integration with a view toward application in survival analysis, The Annals of Statistics 18: 1501-1555.

Glynn, R., Stukel, T., Sharp, S., Bulbolz, T., Freeman, J. e Fisher, E. (1993). Estimating the variance of standardized rates of recurrent events, with application to hospitalizations among the eldery in New England, American Journal of Epidemiology 137: 776-786.

Gopalan, C. (1988). Stunting: significance and implications for public health policy, Linear growth retardation in less develop countries, Raven Press, New York, pp. 265-284.

Greenwood, M. (1926). The natural duration of cancer, Reports of Public Health and Medical Subjects, Vol. 33, Her Majesty's Stationery Office, London.

Hannan, P., Shu, X., Weisdorf, D. e Goldman, A. (1998). Analysis of failure times for multiple infections following bone marrow transplantation: an application of the multiple failure time proportional hazards model, Statistics in Medicine 17: 2371-2380. 
Hoover, D. R. (1996). Extension of the life table to repeating and changing events, American Journal of Epidemiology 143: 1266-1276.

Kalbfleisch, J., Lawless, J. e Vollmer, W. (1983). Estimation in Markov models from aggregate data, Biometrics 39: 907-919.

Karr, A. F. (1986). Point Processes and Their Statistical Inference, Dekker, New York.

Kay, R. (1986). A Markov model for analysing cancer markers and diseases states in survival studies, Biometrics 42: 855-865.

Lawless, J. e Nadeaus, C. (1995). Some simple robust methods for the analysis of recurrent events, Technometrics 37: 158-168.

Lei, D., Chaves, S., Paes, A., Escuder, M., Ribeiro, A., Freire, R. e Lerner, B. (1999). Risk of linear growth retardation during the first two years of life: a new approach, European Journal of Clinical Nutrition 53: 456-460.

Lin, D. (1993). MULCOX2: A general computer program for the Cox regression analysis of multivariate failure time data, Computer Methods and Programs in Biomedicine 40: 279-293.

Lin, D. (1994). Cox regression analysis of multivariate failure time data: The marginal aproach, Statistics in Medicine 13: 2233-2247.

Lin, D. e Wei, L. (1989). The robust inference for the Cox proportional hazards model, Journal of the American Statistical Association 84: 1074-1078.

Marshall, G., Guo, W. e Jones, R. (1995). MARKOV: A computer program for multi-state markov models with covariables, Computer Methods and Programs in Biomedicine 47: 147-156.

Marshall, G. e Jones, R. (1995). Multi-state models and diabetic retinopathy, Statistics in Medicine 14: 1975-1983.

Miller, R. (1974). The Jackknife - a review, Biometrika 61: 1-15.

Murphy, S. (1994). Consistency in a proportional hazards model incorporating a random effect, The Annals of Statistics 22: 712-731. 
Murphy, S. (1995). Asymptotic theory for the frailty model, The Annals of Statistics 23: 182-198.

Ng, E. e Cook, R. (1997). Modeling two-state disease processes with random effects, Lifetime Data Analysis 3: 315-335.

Prentice, R., Williams, B. e Peterson, A. (1981). On the regression analysis of multivariate failure time data, Biometrika 68: 373-379.

Rebolledo, R. (1980). Central limit theorems for local martingales, Z. Wahsch verw. Geb. 51: 269-286.

Rogers, L. C. G. e Williams, D. (1994). Diffusions, Markov Processes and Martingales, Wiley, Chichester.

Staniswallis, J., Thall, P. e Salch, J. (1997). Semiparametric regression analysis for recurrent event interval counts, Biometrics 53: 1334-1353.

Stukel, T., Glynn, R., Fisher, E., Sharph, S., Lu-yao, G. e Wennberg, J. (1994). Standartized rates of recurrent outcomes, Statistics in Medicine 13: 1781-1791.

Thall, P. (1988). Mixed poisson regression models for longitudinal interval count data, Biometrics 44: 197-209.

Thall, P. e Lachin, J. (1988). Analysis of recurrent events: Nonparametric methods for random-interval count data, Journal of the American Statistical Association 83: 339 347.

Therneau, T. e Hamilton, S. (1997). rhdnase as an example of recurrent event analysis, Statistics in Medicine 16: 2029-2047.

Turnbull, B., Jiang, W. e Clark, L. (1997). Regression models for recurrent event data: Parametric random effects models with measurement error, Statistics in Medicine 16: 853-864.

Wei, L. e Glidden, D. (1997). An overview of statistical methods for multiple failure time data in clinical trials, Statistics in Medicine 16: 833-839. 
Wei, L. e Johnson, W. (1985). Combining dependent tests with incomplete repeated measurements, Biometrika 72: 359-364.

Wei, L., Lin, D. e Weissfeld, L. (1989). Regression analysis of multivariate incomplete failure time data by modeling marginal distributions, Journal of the American Statistical Association 84: 1065-1073. 